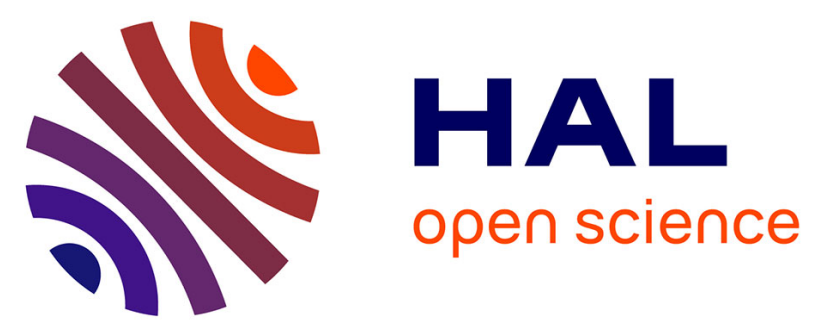

\title{
Extension to various thermal boundary conditions of the elliptic blending model for the turbulent heat flux and the temperature variance
}

\author{
Gaëtan Mangeon, Sofiane Benhamadouche, Jean-François Wald, Remi
}

Manceau

\section{To cite this version:}

Gaëtan Mangeon, Sofiane Benhamadouche, Jean-François Wald, Remi Manceau. Extension to various thermal boundary conditions of the elliptic blending model for the turbulent heat flux and the temperature variance. Journal of Fluid Mechanics, 2020, 905 (A1), pp.1-34. 10.1017/jfm.2020.683 . hal-02974557

\section{HAL Id: hal-02974557 \\ https://hal.inria.fr/hal-02974557}

Submitted on 21 Oct 2020

HAL is a multi-disciplinary open access archive for the deposit and dissemination of scientific research documents, whether they are published or not. The documents may come from teaching and research institutions in France or abroad, or from public or private research centers.
L'archive ouverte pluridisciplinaire HAL, est destinée au dépôt et à la diffusion de documents scientifiques de niveau recherche, publiés ou non, émanant des établissements d'enseignement et de recherche français ou étrangers, des laboratoires publics ou privés.

\section{(ㅇ)(1) $\$$}

Distributed under a Creative Commons Attribution - NonCommercial - NoDerivatives| 4.0 


\title{
Extension to various thermal boundary conditions of the elliptic blending model for the turbulent heat flux and the temperature variance
}

\author{
Gaëtan Mangeon $^{1,2}$, Sofiane Benhamadouche ${ }^{1} \dagger$, Jean-François Wald ${ }^{1}$ \\ and Rémi Manceau ${ }^{2}$ \\ ${ }^{1}$ EDF R\&D, MFEE Dept., 06 quai Watier, 78400 Chatou, France \\ ${ }^{2}$ CNRS, Universite de Pau et des Pays de l'Adour, E2S UPPA, INRIA, project-team CAGIRE, \\ Laboratory of Mathematics and Applied Mathematics (LMAP), Pau, France
}

(Received xx; revised $\mathrm{xx}$; accepted $\mathrm{xx}$ )

A new formulation of the model used in the near-wall region for the turbulent heat flux is developed, in order to extend the Elliptic Blending Differential Flux Model of Dehoux et al., Int. J. Heat Fluid Fl. (2017), to various boundary conditions for the temperature: imposed wall-temperature, imposed heat flux or Conjugate Heat Transfer (CHT). The new model is developed on a theoretical basis in order to satisfy the near-wall budget of the turbulent heat flux and, consequently, its asymptotic behavior in the vicinity of the wall, which is crucial for the correct prediction of heat transfer between the fluid and the wall. The models of the different terms are derived using Taylor series expansions and comparisons with recent direct numerical simulation data of channel flows with various boundary conditions. A priori tests show that this methodology makes it possible to drastically improve the physical representation of the wall/turbulence interaction. This new differential flux model relies on the thermal-to-mechanical time-scale ratio which depends on the thermal boundary condition at the wall. The key element entering this ratio is $\varepsilon_{\theta}$, the dissipation rate of the temperature variance $\overline{\theta^{\prime 2}}$. Thus, a new near-wall model for this dissipation rate is proposed, in the framework of the second-moment closure based on the elliptic-blending strategy. The computations carried out in order to validate the new differential flux model demonstrate the very satisfactory prediction of heat transfer in the forced convection regime for all kinds of thermal boundary condition.

Key words: Turbulence modeling; Turbulent heat flux; Near-wall modeling; Secondmoment closure; Differential Flux Model; Elliptic blending; Temperature variance; Variance dissipation rate

\section{Introduction}

In many industrial applications, the prediction of heat transfer between a fluid and solid walls plays an essential role in design, and in particular in the dimensioning and the selection of materials. On the one hand, a correct estimate of the mean heat transfer is necessary to improve system performance, and on the other hand, the levels of

$\dagger$ Email address for correspondence: sofiane.benhamadouche@edf.fr 
temperature fluctuations in the solid parts are essential to anticipate problems related to thermal fatigue. These issues are particularly sensitive in the field of energy production, in which EDF is a major player, and particularly in nuclear engineering. A reliable estimate of the turbulent heat flux, the mean temperature and its variance is a key issue in order to improve the safety and efficiency of nuclear power plants. A typical example is the case of T-junctions in which hot water and cold water are mixed. Indeed, this situation can induce thermal fatigue and cause serious mechanical damages to the structure, and, in some extreme cases, lead to failure of the pipe walls and fluid leakage. This industrial issue is at the origin of few incidents, such as the case of Civaux nuclear power plant water leak in France in 1998, which occurred in the elbow of a pipe. This issue has gradually gained importance in the literature (see for instance, Howard \& Serre 2015, 2017), which highlights the importance of a detailed prediction of heat transfer, including information about the amplitude of fluctuations.

Most of the industrial applications using computational fluid dynamics (CFD)are still currently treated with simple Eddy-Viscosity Models and the Simple Gradient Diffusion Hypothesis (SGDH) to take into account the turbulent heat flux. One of the most successful advanced approaches in forced convection flows is the elliptic relaxation concept and in particular the $\overline{v^{2}}-f$ model, originally developed by Durbin (1991) (Parneix et al. 1998; Manceau et al. 2000; Billard \& Laurence 2012).

For industrial applications, it is crucial to predict the turbulent heat flux in the wallnormal direction. Indeed, this flux dictates the heat exchanges between the fluid and the wall, and is intimately related to the intensity of the wall-normal velocity fluctuations. This makes Reynolds stress models promising approaches in order to improve the predictions in complex configurations (Hanjalić \& Launder 2011). For a good representation of the near-wall region, the use of wall functions must be avoided (Durbin 1991). Among other models, the Elliptic Blending-Reynolds Stress Model (EB-RSM, Manceau \& Hanjalić 2002; Manceau 2015) have been successfully applied to some heat transfer cases (see, e.g., Angelino et al. 2019; Benhamadouche et al. 2020; Dovizio et al. 2019 for very recent applications). Although the Generalized Gradient Diffusion Hypothesis (GGDH) can be sufficient to model the turbulent heat flux for some applications, it suffers from intrinsic limitations, particularly for complex flows and in the presence of buoyancy effects (Hanjalić 2002). Therefore, in recent years, tremendous efforts have been devoted to the development of differential flux models (DFMs) based on elliptic blending to account for the wall/turbulent heat flux interaction (Choi \& Kim 2008; Shin et al. 2008; Dehoux et al. 2017; Choi et al. 2018).

Most of these DFMs involve the thermal time-scale and thus the dissipation rate of the temperature variance. It is usual to estimate this variable based on the thermalto-mechanical time-scale ratio $R$. This ratio is often set at a constant value (Spalding 1971), which constitutes a reasonable assumption far from the wall. Dehoux et al. (2017) used a variable ratio that tends to the Prandtl number at the wall and asymptotes to a constant value far from the wall. Craft et al. (1996) proposed an algebraic model for $R$ which can deal with free shear flows, including flows influenced by buoyancy. However, these simplified approaches are not valid for all situations, so it is desirable to solve a transport equation for the dissipation rate of the temperature variance. As pointed out by Hanjalić (2002), models using thermal or mixed time scales (for instance, Zeman \& Lumley 1976; Newman et al. 1981; Elghobashi \& Launder 1983; Jones \& Musonge 1988; Abe et al. 1995; Shikazono \& Kasagi 1996) violate the superposition principle. However, as noted by Pope (1983), standard RANS models, which reduce the dynamics of the entire turbulent spectrum to time scales only, cannot both satisfy the superposition principle and correctly represent the observed physics. 
A strong limitation of almost all these models is that their near-wall asymptotic behavior is valid only for the idealized case of vanishing temperature fluctuations, i.e., imposed wall temperature, which corresponds to the boundary condition used in a large majority of the available databases (e.g., Kasagi et al. 1992; Abe et al. 2004). Recently, Tiselj et al. (2001) and Flageul et al. (2015) performed direct numerical simulation (DNS) of turbulent channel flows in the forced convection regime with either an imposed heat flux at the wall or Conjugate Heat Transfer (CHT). They highlighted that the thermal boundary conditions have a major influence on the behavior of turbulent quantities in the near-wall region. Configurations in which temperature fluctuations are non-zero at the wall have been rarely studied by the turbulence modeling community. Notable exceptions are the models developed by Sommer et al. (1994) and Craft et al. (2010), which are both based on the SGDH and wall functions to represent the influence of the wall on the turbulent heat flux.

The motivation for the present work is thus to extend the DFM based on elliptic blending (EB-DFM, Dehoux et al. 2017) to general thermal boundary conditions at the wall. Such an extension will also make possible the future derivation of an elliptic-blending algebraic flux model (EB-AFM, Dehoux 2012), i.e., a simplified approach based on weak equilibrium assumptions for the turbulent heat flux (Hanjalic 2002) that will inherit the improved representation of the influence of the various boundary conditions.

The aim of the present paper is threefold: (i) deriving an improved model for the terms appearing in the turbulent heat flux equation, based on asymptotic arguments, which accommodates all thermal boundary conditions at the wall; (ii) providing a new transport equation for $\varepsilon_{\theta}$, the dissipation rate of the temperature variance, which is a key variable in the temperature variance equation; the modeled equation must also be valid for all thermal boundary conditions at the wall; (iii) validating a posteriori, i.e., by full computations, the new model in the forced convection regime.

In section 2, the thermal models that are used as a starting point are presented for the turbulent heat flux and the temperature variance, with a particular focus on the modeling of the near-wall region using the elliptic blending approach. In section 3 , the asymptotic behavior of the terms involved in the transport equation for the turbulent heat flux is analyzed using Taylor series expansions, depending on the thermal boundary condition at the wall. The last part of this section is devoted to the development and a priori tests of a new turbulent heat flux model that satisfies the asymptotic behavior in the near wall region whatever the boundary conditions. Section 4 is dedicated to the development of a new transport equation for the dissipation rate of the temperature variance which is essential to obtain both an accurate thermal-to-mechanical time-scale ratio and an accurate temperature variance. Finally, in Section 5, the new model is numerically validated against the recent DNS database of Flageul et al. (2015) for a channel flow with three types of wall boundary conditions: an imposed temperature, an imposed heat flux and CHT.

\section{Elliptic Blending Strategy}

Throughout the present article, the instantaneous variables $\phi$ (velocity components $u_{i}$, pressure $p$ or temperature $T$ ) are decomposed into $\phi=\bar{\phi}+\phi^{\prime}$, where $\bar{\phi}$ denotes the Reynolds-averaged variable and $\phi^{\prime}$ its fluctuation.

With respect to the modeling of the Reynolds stresses, the Elliptic-Blending Reynolds Stress Model (EB-RSM) described in Manceau (2015) is used. The main characteristic of this model is that the pressure and dissipation terms, $\phi_{i j}^{*}$ and $\varepsilon_{i j}$, are modeled as a blending of a standard models used far from the walls (denoted with a $h$ exponent) and 
near-wall models (denoted with a $w$ exponent), such that

$$
\phi_{i j}^{*}-\varepsilon_{i j}=\left(1-\alpha^{3}\right)\left(\phi_{i j}^{w}-\varepsilon_{i j}^{w}\right)+\alpha^{3}\left(\phi_{i j}^{h}-\varepsilon_{i j}^{h}\right),
$$

where the blending function $\alpha$ is the solution of the elliptic relaxation equation

$$
\alpha-L^{2} \nabla^{2} \alpha=1 .
$$

Similarly, with regard to the turbulent heat flux $\overline{u_{i}^{\prime} \theta^{\prime}}$ that appears in the mean temperature equation, the baseline model used in the present article is the EllipticBlending Differential Flux Model proposed by Dehoux et al. (2017). The transport equation for the turbulent heat flux reads

$$
\frac{\overline{D u_{i}^{\prime} \theta^{\prime}}}{D t}=P_{\theta i}^{U}+P_{\theta i}^{T}+D_{\theta i}^{\nu+\kappa}+D_{\theta i}^{t}+\phi_{\theta i}^{*}-\varepsilon_{\theta i},
$$

where $P_{\theta i}^{U}, P_{\theta i}^{T}, D_{\theta i}^{\nu+\kappa}, D_{\theta i}^{t}, \phi_{\theta i}^{*}$ and $\varepsilon_{\theta i}$ stand for the production by the mean velocity gradient, the production by the mean temperature gradient, the molecular diffusion, the turbulent diffusion, the scrambling term and the dissipation vector, respectively. The production terms do not require modeling with the present second-moment RANS closures. The turbulent diffusion term is modeled by the GGDH (see Daly \& Harlow 1970) as

$$
D_{\theta i}^{t}=\frac{\partial}{\partial x_{j}}\left(c_{\theta} \overline{u_{j}^{\prime} u_{k}^{\prime}} \tau \frac{\partial \overline{u_{i}^{\prime} \theta^{\prime}}}{\partial x_{k}}\right),
$$

where $\tau$ is Durbin's time scale (Durbin 1991)

$$
\tau=\max \left(\mathcal{T}, C_{T} \sqrt{\frac{\nu}{\varepsilon}}\right) \quad ; \quad \mathcal{T}=\frac{k}{\varepsilon} .
$$

Molecular diffusion is modeled follwing Shikazono \& Kasagi (1996),

$$
D_{\theta i}^{\nu+\kappa}=\frac{\partial}{\partial x_{j}}\left(\frac{\kappa+\nu}{2} \frac{\partial \overline{u_{i}^{\prime} \theta^{\prime}}}{\partial x_{j}}+n_{i} n_{k} \frac{\nu-\kappa}{6} \frac{\partial \overline{u_{k}^{\prime} \theta^{\prime}}}{\partial x_{j}}\right) .
$$

In order for the model to be valid in near-wall regions, the same elliptic blending approach as described above for the Reynolds stress is applied to the difference $\phi_{\theta i}^{*}-\varepsilon_{\theta i}$ (Shin et al. 2008; Choi \& Kim 2008),

$$
\phi_{\theta i}^{*}-\varepsilon_{\theta i}=\left(1-\alpha_{\theta}\right)\left(\phi_{\theta i}^{w}-\varepsilon_{\theta i}^{w}\right)+\alpha_{\theta}\left(\phi_{\theta i}^{h}-\varepsilon_{\theta i}^{h}\right) .
$$

Following Dehoux et al. (2017), $\alpha_{\theta}$ is distinct from the elliptic blending factor $\alpha$ in Eq. (2.1) and is obtained from the additional elliptic equation

$$
\alpha_{\theta}-L_{\theta}^{2} \nabla^{2} \alpha_{\theta}=1,
$$

in which the thermal length scale $L_{\theta}$ is simply related to the dynamic turbulent length scale by $L_{\theta}=2.5 L$ with $L=C_{L} \max \left(\frac{k^{3 / 2}}{\varepsilon}, C_{\eta} \frac{\nu^{3 / 4}}{\varepsilon^{1 / 4}}\right)$. As for $\alpha$, the boundary condition at the wall is $\alpha_{\theta}=0$.

Far from the wall, the standard model of Launder (1988) is used for $\phi_{\theta i}^{*}$, which reads, in the absence of buoyancy,

$$
\phi_{\theta i}^{h}=-C_{1 \theta} \frac{1}{\tau} \overline{u_{i}^{\prime} \theta^{\prime}}+C_{2 \theta} \overline{u_{j}^{\prime} \theta^{\prime}} \frac{\partial \overline{u_{i}}}{\partial x_{j}}
$$

and the dissipation tensor $\varepsilon_{\theta i}^{h}=0$ due to the assumed isotropy of the small scales. The 
near-wall models for $\phi_{\theta i}^{w}$ and $\varepsilon_{\theta i}^{w}$ are built in order to satisfy the near-wall asymptotic behavior of the turbulent heat flux. Dehoux et al. (2017) use

$$
\begin{aligned}
& \phi_{\theta i}^{w}=-\beta \frac{1}{\mathcal{T}}\left[1+C_{w \theta}^{\phi}\left(1-\alpha_{\theta}\right) \frac{P_{k}}{\varepsilon}\right] \overline{u_{j}^{\prime} \theta^{\prime}} n_{i} n_{j}, \\
& \varepsilon_{\theta i}^{w}=C_{\varepsilon}\left[1+C_{w \theta}^{\varepsilon}\left(1-\alpha_{\theta}\right) \frac{P_{k}}{\varepsilon}\right]\left(\frac{\sqrt{P r}}{\sqrt{R} \mathcal{T}} \overline{u_{i}^{\prime} \theta^{\prime}}+\gamma \frac{1}{\mathcal{T}} \overline{u_{j}^{\prime} \theta^{\prime}} n_{i} n_{j}\right),
\end{aligned}
$$

where

$$
\beta=\gamma=\frac{\sqrt{P r}}{\sqrt{R}}
$$

and

$$
C_{\varepsilon}=\frac{1}{2}\left(1+\frac{1}{P r}\right)
$$

and $n_{i}$ is a pseudo-wall-normal vector evaluated as the normalized gradient of $\alpha$. $R$ is the thermal-to-mechanical time-scale ratio

$$
R=\frac{\mathcal{T}_{\theta}}{\mathcal{T}}, \text { where } \quad \mathcal{T}_{\theta}=\frac{\overline{\theta^{\prime 2}}}{2 \varepsilon_{\theta}} \quad \text { and } \quad \mathcal{T}=\frac{k}{\varepsilon} .
$$

It is modeled by

$$
R=\left(1-\alpha_{\theta}\right) \operatorname{Pr}+\alpha_{\theta} R^{h},
$$

where $R^{h}=0.5$ is the value recommended far from the wall for a Prandtl number $\operatorname{Pr}=\nu / \kappa$ around unity (Hanjalić 2002). Dehoux et al. (2017) showed that this model reproduces forced, mixed and natural convection cases a very satisfactory manner compared to simpler approaches for an imposed wall temperature.

As indicated in the introduction, the resolution of $\overline{\theta^{\prime 2}}$ can be important not only for buoyant flows, but also for industrial configurations where thermal fatigue is an issue. The transport equation for the temperature variance reads

$$
\frac{\overline{D \theta^{\prime 2}}}{D t}=2 P_{\theta}-2 \varepsilon_{\theta}+D_{\theta}^{\kappa}+D_{\theta}^{t}
$$

where $P_{\theta}=-\overline{u_{j}^{\prime} \theta^{\prime}} \frac{\partial \bar{\theta}}{\partial x_{j}}$ is the production by the mean temperature gradient and $D_{\theta}^{\kappa}=$ $\frac{\partial}{\partial x_{j}}\left(\kappa \frac{\partial \overline{\theta^{\prime 2}}}{\partial x_{j}}\right)$ the molecular diffusion. For the turbulent diffusion $D_{\theta}^{t}$, the Daly \& Harlow (1970) model is applied

$$
D_{\theta}^{t}=\frac{\partial}{\partial x_{j}}\left(C_{\theta \theta} \tau \overline{u_{i}^{\prime} u_{j}^{\prime}} \frac{\partial \overline{\theta^{\prime 2}}}{\partial x_{j}}\right)
$$

where $\tau$ is Durbin's time scale (2.5).

In Dehoux's model, the dissipation rate of the temperature variance, $\varepsilon_{\theta}$, is evaluated using

$$
\varepsilon_{\theta}=\frac{\overline{\theta^{\prime 2}}}{2 R \mathcal{T}} .
$$

The full set of equations and coefficients is available in Appendix A.

It is important to note that the asymptotic analysis leading to the models for $\phi_{\theta i}^{w}$, $\varepsilon_{\theta i}^{w}$ and $\varepsilon_{\theta}$ is based on the very common assumption that the fluctuations of the wall temperature are negligible due to the thermal inertia of the solid material, such a way 
that a constant temperature can be imposed at the wall. However, in some circumstances, it is crucial to take into account the temperature fluctuations and their propagation in the solid wall (Kasagi et al. 1989). For this reason, Flageul et al. (2015) have recently produced DNS databases in order to identify the different heat transfer characteristics for an imposed wall temperature, an imposed heat flux and CHT. Since the model of Dehoux et al. (2017) presented above was derived using an asymptotic analysis valid for an imposed wall temperature only, the remainder of the present article is devoted to the theoretical analysis and the exploitation of the DNS databases to extend the model to various thermal boundary conditions.

\section{Modeling of the turbulent heat flux}

As mentioned above, the main feature of the original model of Dehoux et al. (2017), simply denoted as Dehoux's model hereafter, is that it is consistent with the asymptotic behavior of near-wall turbulence for an imposed temperature. In order to extend its validity, the asymptotic analysis is generalized to other thermal boundary conditions.

\subsection{Near-wall behavior of the source terms and Dehoux's model limitations}

In the vicinity of a wall located at $y=0$, using the no-slip boundary condition and the divergence-free constraint, it is easy to show that the Taylor series expansions of the fluctuating velocities and temperature with respect to the wall-distance $y$ write

$$
\begin{aligned}
u^{\prime}(x, y, z, t) & =a_{1}(x, z, t) y+a_{2}(x, z, t) y^{2}+\mathcal{O}\left(y^{3}\right) \\
v^{\prime}(x, y, z, t) & =b_{2}(x, z, t) y^{2}+b_{3}(x, z, t) y^{3}+\mathcal{O}\left(y^{4}\right) \\
w^{\prime}(x, y, z, t) & =c_{1}(x, z, t) y+c_{2}(x, z, t) y^{2}+\mathcal{O}\left(y^{3}\right), \\
\theta^{\prime}(x, y, z, t) & =t_{0}(x, z, t)+t_{1}(x, z, t) y+t_{2}(x, z, t) y^{2}+\mathcal{O}\left(y^{3}\right) .
\end{aligned}
$$

The coefficients $a_{k}, b_{k}, c_{k}$ and $t_{k}$ are random variables independent of $y$. In order to alleviate the writing of the expressions, the dependency on $(x, z, t)$ is set aside. The asymptotic behavior of $\overline{u_{i}^{\prime} \theta^{\prime}}, k, \varepsilon, \overline{\theta^{\prime 2}}$ and $\varepsilon_{\theta}$ are easily deduced:

$$
\begin{aligned}
& \overline{u^{\prime} \theta^{\prime}}=\overline{a_{1} t_{0}} y+\left(\overline{a_{1} t_{1}}+\overline{a_{2} t_{0}}\right) y^{2}+\mathcal{O}\left(y^{3}\right), \\
& \overline{v^{\prime} \theta^{\prime}}=\overline{b_{2} t_{0}} y^{2}+\left(\overline{b_{2} t_{1}}+\overline{b_{3} t_{0}}\right) y^{3}+\mathcal{O}\left(y^{4}\right) \text {, } \\
& \overline{w^{\prime} \theta^{\prime}}=\overline{c_{1} t_{0}} y+\left(\overline{c_{1} t_{1}}+\overline{c_{2} t_{0}}\right) y^{2}+\mathcal{O}\left(y^{3}\right) \text {, } \\
& k=\frac{1}{2} \overline{u_{i}^{\prime} u_{i}^{\prime}}=\frac{1}{2}\left(\overline{a_{1}^{2}}+\overline{c_{1}^{2}}\right) y^{2}+\mathcal{O}\left(y^{3}\right), \\
& \varepsilon=\nu \overline{\frac{\partial u_{i}^{\prime}}{\partial x_{k}} \frac{\partial u_{i}^{\prime}}{\partial x_{k}}}=\nu\left(\overline{a_{1}^{2}}+\overline{c_{1}^{2}}\right)+\mathcal{O}(y), \\
& \overline{\theta^{\prime 2}}=\overline{t_{0}^{2}}+2 \overline{t_{0} t_{1}} y+\left(\overline{t_{1}^{2}}+2 \overline{t_{0} t_{2}}\right) y^{2}+\mathcal{O}\left(y^{3}\right) \text {, } \\
& \varepsilon_{\theta}=\kappa \overline{\frac{\partial \theta^{\prime}}{\partial x_{k}} \frac{\partial \theta^{\prime}}{\partial x_{k}}}=\kappa\left[\overline{\left(\frac{\partial t_{0}}{\partial x}\right)^{2}}+\overline{t_{1}^{2}}+\overline{\left(\frac{\partial t_{0}}{\partial z}\right)^{2}}\right]+\kappa\left[2 \overline{\frac{\partial t_{0}}{\partial x} \frac{\partial t_{1}}{\partial x}}+4 \overline{t_{1} t_{2}}+2 \frac{\partial t_{0}}{\partial z} \frac{\partial t_{1}}{\partial z}\right] y \\
& +\kappa\left[\overline{\left(\frac{\partial t_{1}}{\partial x}\right)^{2}}+2 \overline{\frac{\partial t_{0}}{\partial x} \frac{\partial t_{2}}{\partial x}}+4 \overline{t_{2}^{2}}+6 \overline{t_{1} t_{3}}+\overline{\left(\frac{\partial t_{1}}{\partial z}\right)^{2}}+2 \overline{\frac{\partial t_{0}}{\partial z} \frac{\partial t_{2}}{\partial z}}\right] y^{2}+\mathcal{O}\left(y^{3}\right) .
\end{aligned}
$$




\begin{tabular}{cccccc}
\hline & $P_{\theta i}^{U}+P_{\theta i}^{T}$ & $\phi_{\theta i}$ & $D_{\theta i}^{\nu+\kappa}$ & $\varepsilon_{\theta i}$ & $D_{\theta i}^{t}$ \\
\hline \hline Imposed temperature & $\mathcal{O}\left(y^{3}\right)$ & $\mathcal{O}(y)$ & $\mathcal{O}(1)$ & $\mathcal{O}(1)$ & $\mathcal{O}\left(y^{3}\right)$ \\
\hline Imposed heat flux & $\mathcal{O}\left(y^{2}\right)$ & $\mathcal{O}(1)$ & $\mathcal{O}(1)$ & $\mathcal{O}(y)$ & $\mathcal{O}\left(y^{2}\right)$ \\
\hline Conjugate Heat Transfer (CHT) & $\mathcal{O}\left(y^{2}\right)$ & $\mathcal{O}(1)$ & $\mathcal{O}(1)$ & $\mathcal{O}(1)$ & $\mathcal{O}\left(y^{2}\right)$ \\
\hline
\end{tabular}

TABLE 1. Asymptotic behavior of the source terms appearing in the exact transport equations for $\overline{u^{\prime} \theta^{\prime}}$ and $\overline{w^{\prime} \theta^{\prime}}$.

The leading order terms of the Taylor series expansions of the thermal variables depend on the temperature boundary condition. For an imposed temperature, $\theta^{\prime}$ goes to zero at the wall, and therefore $t_{0}=0$. For an imposed heat flux, $t_{0} \neq 0$, but the derivative of $\theta^{\prime}$ in the wall normal direction is zero, so that $t_{1}=0$. Finally, for CHT, there is no specific simplification: $t_{0}$ and $t_{1}$ can take any value.

The asymptotic behavior of the exact source terms of the turbulent heat flux transport equations can then be determined using the Taylor series expansions presented above. It is not necessary to detail the calculations here, and only the final results are presented in Table 1 for the stream-wise and span-wise components and in Table 2 for the wall-normal component. Three important observations can be made on the basis of these tables. (i) The behavior of the terms of the transport equations for $\overline{u^{\prime} \theta^{\prime}}$ and $\overline{w^{\prime} \theta^{\prime}}$ is the same, as well as their expansions in Eq. (3.2), so that it is not necessary to detail the behavior of both components: in the rest of this paper, only the component $\overline{u^{\prime} \theta^{\prime}}$ is considered. (ii) The asymptotic behavior of these exact source terms depends on the thermal boundary condition to such an extent that the terms appearing at leading order are different. For instance, for the wall-normal component (Table 2), the dissipation term $\varepsilon_{\theta i}$ is a dominant term for an imposed temperature, whereas it is negligible in the other two cases. (iii) The production terms $P_{\theta i}^{U}$ and $P_{\theta i}^{T}$ and the turbulent diffusion term $D_{\theta i}^{t}$ are always negligible compared to the molecular diffusion $D_{\theta i}^{\nu+\kappa}$, the scrambling $\phi_{\theta i}$ and the dissipation $\varepsilon_{\theta i}$ terms; consequently it is sufficient to consider the last three terms in the asymptotic analysis of the transport equation for the turbulent heat flux. Therefore, the analysis focuses on the near-wall balance

$$
D_{\theta i}^{\nu+\kappa}+\phi_{\theta i}-\varepsilon_{\theta i}=0
$$

that must be satisfied for all the cases.

Dehoux's model was derived in order to satisfy (3.7) in the case of an imposed temperature at the wall. One of the limitations of this model is that it makes use of the algebraic expression (2.15) to represent the thermal-to-mechanical time-scale ratio $R$, in order to avoid the resolution of a transport equation for the dissipation rate of the variance, $\varepsilon_{\theta}$. As shown is Tab. 3, the wall limiting behavior of $R$ strongly depends on the thermal boundary condition: the exact $R$ goes to the Prandtl number at the wall for an imposed temperature, while it tends to infinity in the other two cases. However, in all cases, the algebraic model (2.15) tends to the Prandtl number. This situation is 


\begin{tabular}{cccccc}
\hline & $P_{\theta i}^{U}+P_{\theta i}^{T}$ & $\phi_{\theta i}$ & $D_{\theta i}^{\nu+\kappa}$ & $\varepsilon_{\theta i}$ & $D_{\theta i}^{t}$ \\
\hline \hline Imposed temperature & $\mathcal{O}\left(y^{4}\right)$ & $\mathcal{O}(y)$ & $\mathcal{O}(y)$ & $\mathcal{O}(y)$ & $\mathcal{O}\left(y^{4}\right)$ \\
\hline Imposed heat flux & $\mathcal{O}\left(y^{3}\right)$ & $\mathcal{O}(1)$ & $\mathcal{O}(1)$ & $\mathcal{O}\left(y^{2}\right)$ & $\mathcal{O}\left(y^{3}\right)$ \\
\hline Conjugate Heat Transfer (CHT) & $\mathcal{O}\left(y^{3}\right)$ & $\mathcal{O}(1)$ & $\mathcal{O}(1)$ & $\mathcal{O}(y)$ & $\mathcal{O}\left(y^{3}\right)$ \\
\hline
\end{tabular}

TABLE 2. Asymptotic behavior of the source terms appearing in the exact transport equation for $\overline{v^{\prime} \theta^{\prime}}$.

\begin{tabular}{lccc}
\hline $\begin{array}{c}\text { Imposed } \\
\text { temperature }\end{array}$ & $\begin{array}{c}\text { Imposed } \\
\text { heat flux }\end{array}$ & Conjugate Heat Transfer (CHT) \\
\hline \hline Exact $R$ & $\nu / \kappa=P r$ & $\mathcal{O}\left(1 / y^{2}\right)$ & $\mathcal{O}\left(1 / y^{2}\right)$ \\
\hline
\end{tabular}

TABLE 3. Asymptotic behavior of the exact expression of the thermal-to-mechanical time-scale ratio $R$.

illustrated by Fig. 1, which shows an a priori evaluation of this algebraic model for $R$; the elliptic equation (2.8) is solved by using the length scale computed from the DNS data, in order to evaluate the value of $R$ given by the algebraic relation (2.15), which is independent of the thermal boundary condition. The comparison made in Fig. 1 with the exact value of $R$ given by Eq. (2.14) extracted from the DNS databases shows that this model for $R$ is not sufficiently general, since the near-wall behavior of the model is not sensitive to the thermal boundary condition.

The main consequence of this lack of generality of the model for $R$ is that the models (2.10) and (2.11) for the scrambling term $\phi_{\theta i}$ and the dissipation term $\varepsilon_{\theta i}$, respectively, are not compatible with an imposed heat flux or CHT. This can be observed in Figs. 2 and 3 for $\varepsilon_{\theta i}$, and in Fig. 4 for $\phi_{\theta i}$. The scrambling term is only shown for CHT as the profiles are very similar with an imposed heat flux. For the tangential component $\varepsilon_{\theta 1}$ of the dissipation term, the problem comes from the near-wall contribution $\varepsilon_{\theta 1}^{w}$ given by Eq. (2.11), which goes to infinity at the wall as $1 / y$, while the asymptotic analysis of the exact term shows that it goes to zero as $y$ for an imposed flux and to a constant for CHT (Table 1). In contrast, for $\phi_{\theta 1}$, the near-wall contribution $\phi_{\theta 1}^{w}$ is correct, but its behavior is strongly perturbed by the component $\phi_{\theta 1}^{h}$ given by Eq. (2.9) that goes to infinity at the wall and is not sufficiently damped by the factor $\alpha_{\theta}$ in Eq. (2.7). Moreover, although the situation seems less critical for the normal component of the dissipation term, its behavior is also not correct as it tends towards a non-zero value at the wall. Therefore, in order to derive a model valid for an imposed heat flux and CHT as 


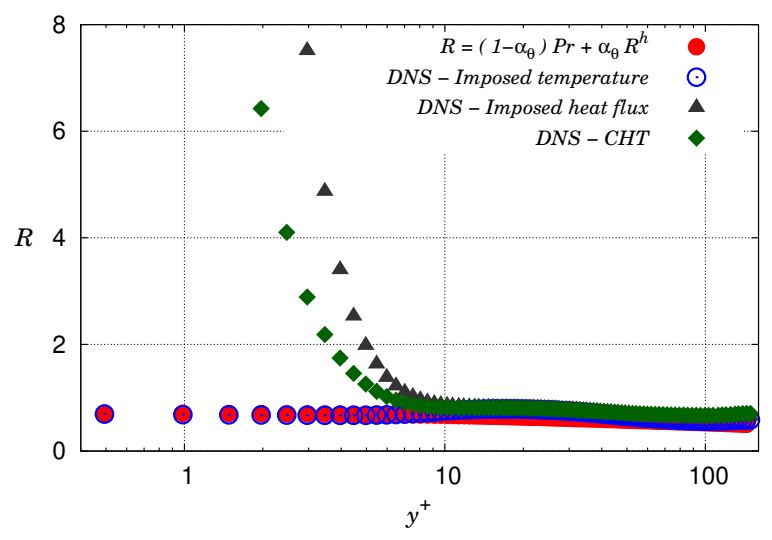

FiguRE 1. Thermal-to-mechanical time-scale ratio $R$ for different temperature boundary conditions. Channel flow DNS data of Flageul et al. (2015). For the CHT case, the solid and the fluid have the same thermal conductivity and diffusivity.

well as for an imposed wall temperature, the asymptotic analysis of Dehoux et al. (2017) must be extended to various thermal boundary conditions, which is done in section 3.2.

\subsection{Asymptotic behavior of $\overline{u_{i}^{\prime} \theta^{\prime}}$ with the right asymptotic behavior of $R$}

In the previous section, it has been concluded that the validity of Dehoux's model is limited to the case of an imposed temperature, mainly due to the model (2.15) for $R$, which is not sufficiently general. However, the following question must also be raised: would correcting the expression for $R$ be sufficient for the model to give the right asymptotic behavior of turbulent heat flux? We will see in the present section that the answer is no.

The procedure is as follows: it is assumed that the exact asymptotic behavior of the thermal-to-mechanical time-scale ratio $R$ is respected in all the cases; the near-wall behavior of the solution of the modeled transport equation for the turbulent heat flux is analyzed and compared with the exact behavior, in order to identify how the model can be made more general.

If one uses the exact asymptotic behavior of $R$, Dehoux's model yields $\varepsilon_{\theta 1}^{w}=\mathcal{O}(1)$ and $\varepsilon_{\theta 2}^{w}=\mathcal{O}(y)$ whatever the temperature boundary condition at the wall. By comparing with Tables 1 and 2, it can be seen that the model is correct for both an imposed temperature condition and CHT. However, for an imposed heat flux, the near-wall behavior of $\varepsilon_{\theta i}^{w}$ is one order below that of the exact term. After considering several options, we have not found any solution to modify the behavior in this case without spoiling the behavior in the other two cases. Therefore, our strategy consists in keeping the near-wall model $\varepsilon_{\theta i}^{w}$ proposed by Dehoux et al. (2017), and to account for the discrepancy with the exact behavior in the case of imposed heat flux in the derivation of the model $\phi_{\theta i}^{w}$. Indeed, as mentioned above, as long as the near-wall balance between the molecular diffusion $D_{\theta i}^{\nu+\kappa}$, the scrambling term $\phi_{\theta i}$ and the dissipation term $\varepsilon_{\theta i}$ is satisfied, the solution of the transport equation for the turbulent heat flux will have the correct asymptotic behavior.

Dehoux's model for $\varepsilon_{\theta i}^{w}$ is associated to the model of Shikazono \& Kasagi (1996) for $D_{\theta i}^{\nu+\kappa}$ (Eq. 2.6). The model $\phi_{\theta i}^{w}$ must be derived in such a way that the correct nearwall balance (3.7) is ensured. As demonstrated below, this can be achieved simply by modifying the coefficient $\beta$ in Eq. (2.10). 

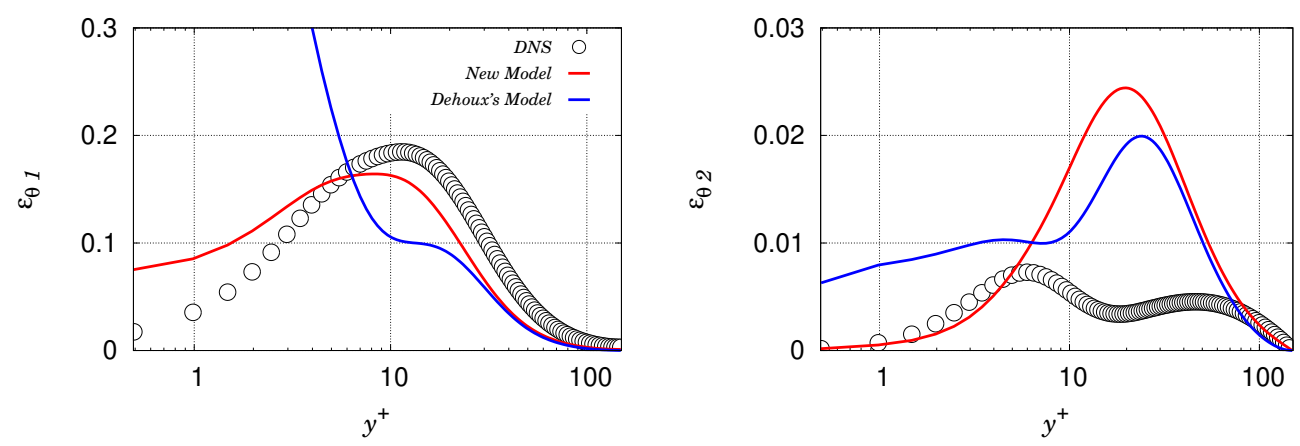

Figure 2. A priori evaluation of the dissipation term for an imposed heat flux. Left: stream-wise component; Right: wall-normal component. Channel flow DNS data of Flageul et al. (2015).
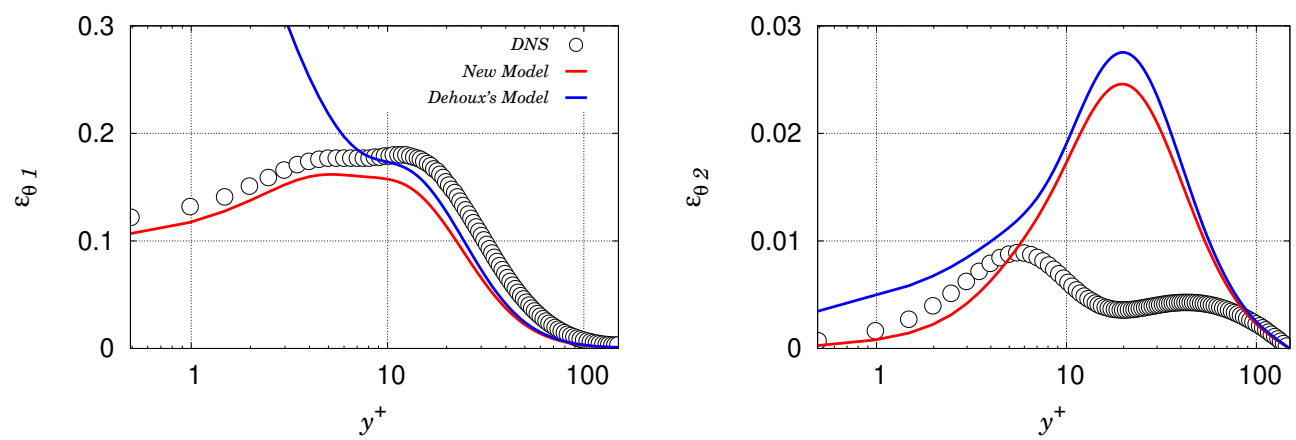

Figure 3. A priori evaluation of the dissipation term for Conjugate Heat Transfer (CHT). Left: stream-wise component; Right: wall-normal component. Channel flow DNS data of Flageul et al. (2015).
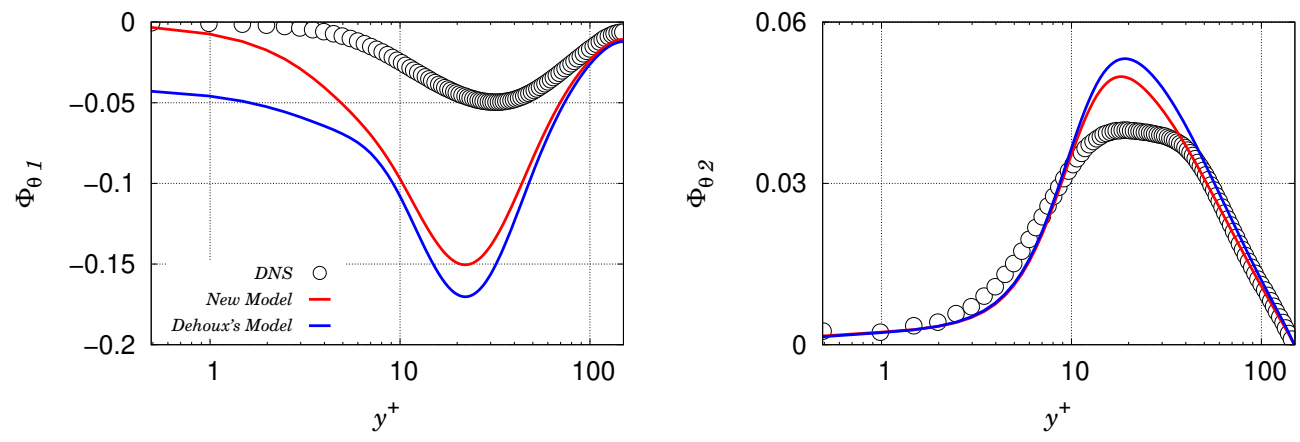

Figure 4. A priori evaluation of the scrambling term for Conjugate Heat Transfer (CHT). Left: stream-wise component; Right: wall-normal component. Channel flow DNS data of Flageul et al. (2015).

In order to determine the relevant value of $\beta$, it is necessary to analyse the asymptotic behavior of the solution of the transport equation for the turbulent heat flux, following the same methodology as proposed by Durbin (1991) for the Reynolds-stress tensor. Considering only the dominant terms in the near-wall region (Eq. 3.7), solutions are 
sought under the form of Taylor series

$$
\overline{u^{\prime} \theta^{\prime}}=A_{1} y+B_{1} y^{2}+C_{1} y^{3}+\mathcal{O}\left(y^{4}\right)
$$

and

$$
\overline{v^{\prime} \theta^{\prime}}=A_{2} y+B_{2} y^{2}+C_{2} y^{3}+\mathcal{O}\left(y^{4}\right) .
$$

Introducing these Taylor series into Eq. (3.7) leads to constraints that the expansion coefficients $A_{i}, B_{i}$ and $C_{i}$ must satisfy, and, consequently, the asymptotic behavior of the solutions can be determined. The model $\phi_{\theta i}^{w}$ must be formulated in order for these solutions to match the exact asymptotic behavior of the turbulent heat flux.

Using the models $(2.6),(2.10)$ and $(2.11)$ for $D_{\theta i}^{\nu+\kappa}, \varepsilon_{\theta i}^{w}$ and $\phi_{\theta i}^{w}$, respectively, the nearwall balance (3.7) writes

$$
\frac{\nu+\kappa}{2} \frac{\partial^{2} \overline{u^{\prime} \theta^{\prime}}}{\partial y^{2}}+\frac{\nu+\kappa}{2}\left(\frac{\partial^{2} \overline{u^{\prime} \theta^{\prime}}}{\partial x^{2}}+\frac{\partial^{2} \overline{u^{\prime} \theta^{\prime}}}{\partial z^{2}}\right)=(\nu+\kappa) \psi \sqrt{\frac{1}{2 \nu \mathcal{T}} \overline{u^{\prime} \theta^{\prime}}}
$$

and

$$
\begin{aligned}
&\left(\frac{2}{3} \nu+\frac{1}{3} \kappa\right) \frac{\partial^{2} \overline{v^{\prime} \theta^{\prime}}}{\partial y^{2}}+\left(\frac{2}{3} \nu+\frac{1}{3} \kappa\right)\left(\frac{\partial^{2} \overline{v^{\prime} \theta^{\prime}}}{\partial x^{2}}+\frac{\partial^{2} \overline{v^{\prime} \theta^{\prime}}}{\partial z^{2}}\right)= \\
& 2(\nu+\kappa) \psi \sqrt{\frac{1}{2 \nu \mathcal{T}} \overline{v^{\prime} \theta^{\prime}}+\beta \frac{1}{\mathcal{T}} \overline{v^{\prime} \theta^{\prime}}}
\end{aligned}
$$

where

$$
\psi=\sqrt{\frac{\varepsilon_{\theta}}{\kappa \overline{\theta^{\prime 2}}}} .
$$

These equations can be simplified based on: the expression (2.13) for $C_{\varepsilon}$; the Taylor series expansion $1 / \mathcal{T}=2 \nu / y^{2}\left(1+a_{\mathcal{T}} y+b_{\mathcal{T}} y^{2}+\mathcal{O}\left(y^{3}\right)\right)$ deduced from the Taylors series expansions of the turbulent kinetic energy $k$ and its dissipation rate $\varepsilon$ (Eqs. (3.3) and (3.4)); the fact that the term $\left(1-\alpha_{\theta}\right) \frac{P_{k}}{\varepsilon}$ that appears in the models $\varepsilon_{\theta i}^{w}$ and $\phi_{\theta i}^{w}$ behaves as $y^{3}$ in the vicinity of the wall; and the general forms of the solutions (3.8) and (3.9). Eqs. (3.10) and (3.11) then become

$$
\begin{gathered}
(\nu+\kappa) B_{1}+\left(3(\nu+\kappa) C_{1}+\chi_{A_{1}}\right) y+\mathcal{O}\left(y^{2}\right)= \\
(\nu+\kappa) \psi\left[A_{1}+\left(B_{1}+\frac{1}{2} a_{\mathcal{T}} A_{1}\right) y+\left(\frac{1}{2} b_{\mathcal{T}} A_{1}+\frac{1}{2} a_{\mathcal{T}} B_{1}+C_{1}\right) y^{2}+\mathcal{O}\left(y^{2}\right)\right]
\end{gathered}
$$

and

$$
\begin{gathered}
\left(\frac{4}{3} \nu+\frac{2}{3} \kappa\right) B_{2}+\left((4 \nu+2 \kappa) C_{2}+\chi_{A_{2}}\right) y+\mathcal{O}\left(y^{2}\right)= \\
(\nu+\kappa) \psi\left[A_{2}+\left(B_{2}+\frac{1}{2} a_{\mathcal{T}} A_{2}\right) y+\left(\frac{1}{2} b_{\mathcal{T}} A_{2}+\frac{1}{2} a_{\mathcal{T}} B_{2}+C_{2}\right) y^{2}+\mathcal{O}\left(y^{2}\right)\right] \\
2 \nu \beta\left[\frac{A_{2}}{y}+\left(B_{2}+a_{\mathcal{T}} A_{2}\right)+\left(b_{\mathcal{T}} A_{2}+a_{\mathcal{T}} B_{2}+C_{2}\right) y+\mathcal{O}(y)\right] . \\
\text { where } \chi_{A_{1}}=\frac{\nu+\kappa}{2}\left(\frac{\partial^{2} A_{1}}{\partial x^{2}}+\frac{\partial^{2} A_{1}}{\partial z^{2}}\right) \text { and } \chi_{A_{2}}=\left(\frac{2}{3} \nu+\frac{1}{3} \kappa\right)\left(\frac{\partial^{2} A_{2}}{\partial x^{2}}+\frac{\partial^{2} A_{2}}{\partial z^{2}}\right) .
\end{gathered}
$$


In order to obtain this development up to order 1 and noticing that the lowest order of $\overline{u^{\prime} \theta^{\prime}}$ and $\overline{v^{\prime} \theta^{\prime}}$ is 1 , the Taylor series expansion of $1 / \mathcal{T}$ had to be carried out up to order 2 as $\psi$ might introduce a term which behaves as $1 / y$ at the wall.

As the Taylor series expansion of $\psi$ depends on the thermal boundary condition, two cases need to be considered separately to obtain relations or constraints on the coefficients $A_{i}, B_{i}$ and $C_{i}$; on the one hand, an imposed temperature at the wall and on the other hand, an imposed heat flux or CHT.

\subsubsection{Imposed wall temperature}

The case of an imposed wall temperature is considered first, for which $t_{0}=0$. Using the Taylor series expansions of $\overline{\theta^{\prime 2}}$ and $\varepsilon_{\theta}$ given by Eqs. (3.5) and (3.6), respectively, it is found that $\psi=1 / y\left(1+a_{\psi} y+b_{\psi} y^{2}+\mathcal{O}\left(y^{3}\right)\right)$, such that for the stream-wise component, Eq. (3.13) writes

$$
(\nu+\kappa) B_{1}+\mathcal{O}(y)=(\nu+\kappa) \frac{A_{1}}{y}+(\nu+\kappa)\left[\left(\frac{1}{2} a_{\mathcal{T}}+a_{\psi}\right) A_{1}+B_{1}\right]+\mathcal{O}(y)
$$

For function (3.8) to be solution of Eq. (3.15), the two sides of Eq. (3.15) must balance at each order $y^{n}$. Balancing the terms at the orders $n=-1$ and $n=0$ yields

$$
\begin{aligned}
0 & =(\nu+\kappa) A_{1} \\
(\nu+\kappa) B_{1} & =(\nu+\kappa)\left[\left(\frac{1}{2} a_{\mathcal{T}}+a_{\psi}\right) A_{1}+B_{1}\right] .
\end{aligned}
$$

The first relation shows that $A_{1}=0$. With $A_{1}=0$, the second relation shows that there is no particular constraint on $B_{1}$ since any value satisfies this equation. In conclusion, any solution $\overline{u^{\prime} \theta^{\prime}}$ of Eq. (3.10) is of the form $\overline{u^{\prime} \theta^{\prime}}=B_{1} y^{2}+\mathcal{O}\left(y^{3}\right)$, which is the expected behavior for an imposed temperature at the wall. As mentioned above, this conclusion holds for $\overline{w^{\prime} \theta^{\prime}}$ as well, i.e., $\overline{w^{\prime} \theta^{\prime}}=B_{3} y^{2}+\mathcal{O}\left(y^{3}\right)$.

For the wall-normal component $\overline{v^{\prime} \theta^{\prime}}$, considering that $\beta$ goes to 1 at the wall as in Dehoux's algebraic model (see Eq. (2.15)), Eq. (3.14) leads, after similar algebraic manipulations, to consider the terms from order $n=-1$ up to order $n=1$ (note that the second order term in the Taylor series expansion of $\psi$ must be taken into account for this component).

The orders $n=-1$ and $n=0$ yield $A_{2}=B_{2}=0$, respectively. The order $n=1$ gives, using the fact that $\beta$ tends to a constant value at the wall, $(4 \nu+2 \kappa)\left(C_{2}+\chi_{A_{2}}\right)=$ $(4 \nu+2 \kappa) C_{2}$. Since $A_{2}=0, \chi_{A_{2}}$ is zero as well, and it is seen that any value of $C_{2}$ satisfies the relation. Therefore, any solution $\overline{v^{\prime} \theta^{\prime}}$ of the equation is of the form $\overline{v^{\prime} \theta^{\prime}}=$ $C_{2} y^{3}+\mathcal{O}\left(y^{4}\right)$, as expected for this component.

\subsubsection{Imposed wall heat flux and Conjugate Heat Transfer}

As mentioned above, the case of an imposed heat flux ( $t_{1}$ is zero) and the case of CHT $\left(t_{0}\right.$ and $t_{1}$ are both non-zero) can be treated simultaneously. Indeed, the asymptotic behavior of $\psi$ obtained using Eqs. (3.5) and (3.6) is in both cases to $\psi=\mathcal{O}(1)$. The objective is again to obtain the expected asymptotic behavior at the wall $\overline{u^{\prime} \theta^{\prime}}=\mathcal{O}(y)$ and $\overline{v^{\prime} \theta^{\prime}}=\mathcal{O}\left(y^{2}\right)$. As $\psi$ goes to a constant at the wall, it is enough here to use the Taylor series expansion of $\psi$ up to order 1: $\psi=\psi_{0}+a_{\psi}^{\prime} y+\mathcal{O}\left(y^{2}\right)$. 
After some algebraic manipulations, it is obtained that, for the stream-wise component, it is sufficient to consider the order $n=0$, which leads to $(\nu+\kappa) B_{1}=(\nu+\kappa) \psi_{0} A_{1}$. Since $\psi_{0} \neq 0$, this equation imposes a relation between $A_{1}$ and $B_{1}$, without constraining the value of $A_{1}$ to be zero. Therefore, $\overline{u^{\prime} \theta^{\prime}}=\mathcal{O}(y)$, which is the expected behavior of the solutions. The analysis is valid for $\overline{w^{\prime} \theta^{\prime}}$ as well, i.e., $\overline{w^{\prime} \theta^{\prime}}=\mathcal{O}(y)$. For the wall-normal component, at orders $n=-1$ and $n=0$, Eq. (3.11) yields

$$
\begin{aligned}
0 & =2 \beta \nu A_{2}, \\
\frac{1}{3}(4 \nu+2 \kappa) B_{2} & =2(\nu+\kappa) \psi_{0} A_{2}+2 \nu \beta\left(a_{\mathcal{T}} A_{2}+B_{2}\right),
\end{aligned}
$$

respectively. The first relation gives $A_{2}=0$ as far as $\beta \neq 0$. We have reached the point where we must make the right choice for $\beta$ in order to impose $\overline{v^{\prime} \theta^{\prime}}=\mathcal{O}\left(y^{2}\right): \beta$ must be chosen in such a way that the second line of Eq. (3.17) is satisfied for any non zero value of $B_{2}$. The equation that must be satisfied is

$$
\beta=\frac{2}{3}+\frac{1}{3} \frac{1}{P r}
$$

or, more generally, $\beta$ must asymptotes to this value at the wall in the case of an imposed heat flux or CHT.

This section illustrates the fact that Dehoux's model, with $\beta=\sqrt{\operatorname{Pr}} / \sqrt{R}$, designed for an imposed wall temperature, is not valid for an imposed heat flux and CHT, even if a correct model for $R$ is used, as $\beta$ tends to 0 with this formulation. In order to extend the validity of the model, the $\beta$ coefficient, which enters the near-wall model $\phi_{\theta i}^{w}$, must tend to 1 at the wall for an imposed temperature and to $2 / 3+1 /(3 P r)$ for the other two cases. The next section is devoted to the derivation of such a model.

\subsection{A new model for the scrambling term and a priori tests}

In the present section, Dehoux's model is extended to various thermal boundary conditions, following the findings of the asymptotic analysis performed in the previous section. The model is first derived, then an a priori evaluation of the model using the DNS databases of Flageul et al. (2015) is performed.

As concluded in the previous section, the model must be able to naturally distinguish the type of the thermal boundary condition at the wall in order to adapt the limiting behavior of the $\beta$ coefficient. Now, to write a general model, i.e., a model that is expressed in the same form in all configurations, it is necessary to involve in its expression one or several quantities that are sensitive to boundary conditions. Only quantities that are solutions of a second order differential equation exhibit this property. In the context of elliptic blending, it is tempting to rely on the $\alpha_{\theta}$ variable, whose wall boundary condition could be defined differently depending on the thermal boundary condition at the same wall. Unfortunately, $\alpha_{\theta}$ is involved in different terms of the model and such an approach would skew the asymptotic behaviour of these terms. We have come to the conclusion that the only way to sensitize the model to thermal boundary conditions is to involve a dimensionless parameter dependent on $\overline{\theta^{\prime 2}}, \varepsilon_{\theta}$ or both. Thus, the key parameter that enters the model is the thermal-to-mechanical time-scale ratio $R=\mathcal{T}_{\theta} / \mathcal{T}$. Indeed, Fig. 1 and Table 3 show that $R$ tends to the Prandtl number at the wall for an imposed temperature and to infinity in other cases. Consequently, the ratio $\sqrt{\mathrm{Pr}} / \sqrt{R}$ is used in the expression for $\beta$ to sensitize the model to the type of wall thermal boundary condition. The proposed expression is

$$
\beta=\frac{2}{3}+\frac{1}{3} \frac{1}{P r}+\frac{1}{3} \frac{\sqrt{P r}}{\sqrt{R}}\left(1-\frac{1}{P r}\right),
$$


such that $\beta$ tends to 1 for an imposed temperature $(R \rightarrow \operatorname{Pr})$ and to $2 / 3+1 /(3 \operatorname{Pr})$ for the two other thermal boundary conditions $(R \rightarrow \infty)$. In contrast, as mentioned above, $\gamma$ in Eq. (2.11) does not need to be modified, and the expression of Dehoux et al. (2017),

$$
\gamma=\frac{\sqrt{P r}}{\sqrt{R}}
$$

is used. With this simple but decisive modification of the model, the near-wall balance between the molecular diffusion $D_{\theta i}^{\nu+\kappa}$, the scrambling $\phi_{\theta i}$ and the dissipation $\varepsilon_{\theta i}$ terms, Eq. (3.7), is respected in the near-wall region, which is the necessary condition for reproducing the adequate asymptotic behavior of the turbulent heat flux components, as demonstrated in section 3.2 .

It is worth noting again that, for this model to be valid for the three types of wall boundary conditions, it is necessary for the thermal-to-mechanical time-scale ratio $R$ to have the right limiting behavior at the wall, which will require solving a transport equation for the temperature variance $\overline{\theta^{\prime 2}}$ and its dissipation $\varepsilon_{\theta}$, rather than using a simple algebraic relation such as Eq. (2.15). In particular, a model for $\varepsilon_{\theta}$, asymptotically correct for all types of thermal boundary conditions, is required. Therefore, a specific model has been developed, which is presented in section 4 .

One could legitimately point out that solving additional transport equations for $\overline{\theta^{\prime 2}}$ and $\varepsilon_{\theta}$ is particularly costly when it is simply a matter of sensitizing the model to thermal boundary conditions. On the one hand, as noted above, only a nondimensional parameter constructed from these variables can define a sufficiently general model. On the other hand, it is important to remember that, as mentioned in the introduction, one of the main interests in developing a model valid in CHT is to be able to estimate thermal fatigue from the temperature variance in the solid. It is therefore necessary to solve the equation of $\overline{\theta^{\prime 2}}$, but also of $\varepsilon_{\theta}$, since, as will be shown in section 4 , the asymptotic behavior of $\varepsilon_{\theta}$ is complex and has a strong influence on that of $\overline{\theta^{\prime 2}}$.

A priori tests are carried out using recent DNS data of Flageul et al. (2015) of a channel flow in the forced convection regime, for different boundary conditions: imposed temperature, imposed heat flux and CHT. The flow is driven by a pressure gradient in the stream-wise direction. The temperature is considered as a passive scalar and the flow is periodic in the stream-wise and span-wise directions. The test-case is defined by two non-dimensional numbers, the friction Reynolds number $R e_{\tau}=\delta u_{\tau} / \nu=149$ and the Prandtl number $\operatorname{Pr}=0.71$. When the solid part is resolved in the DNS computations, the continuity of the heat flux is imposed at the fluid-solid interface. Note that the CHT case considered herein is the case considered by Flageul et al. (2015), where the solid and the fluid have the same thermal conductivity and diffusivity and thus the dissipation rate of the temperature variance is continuous at the fluid-solid interface. Reproducing this case is already a big challenge, and is sufficient to validate the new model for the turbulent heat flux since its asymptotic behavior is not affected by these physical properties.

The case of an imposed temperature at the wall is considered first. Figure 5 compares with DNS data the sum of the molecular diffusion, the scrambling and the dissipation terms obtained with Dehoux's model and the new model. As mentioned above, these terms are dominant in the near-wall budget and determine the asymptotic behavior of the turbulent heat flux components. It can be seen that the new model correctly reproduces the balance of the three terms in the case of an imposed temperature at the wall. The results given by the new model are slightly less accurate than those given by Dehoux's model in the buffer layer, but this is not a significant problem since, in this region, the budgets of the turbulent heat flux components are dominated by the production 

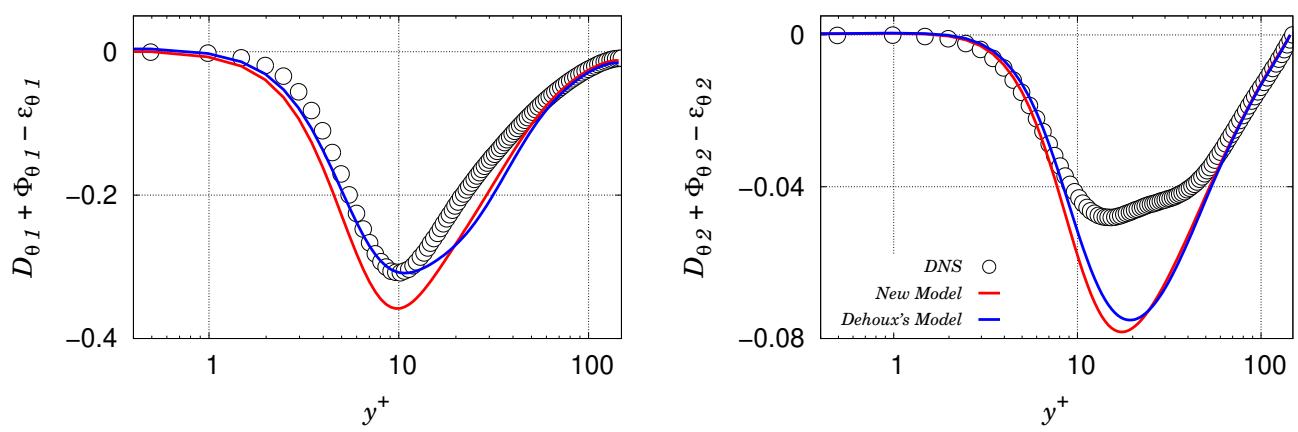

FiguRE 5. Sum of the molecular diffusion, the scrambling and the dissipation terms for an imposed temperature. Left: stream-wise component; Right: wall-normal component.
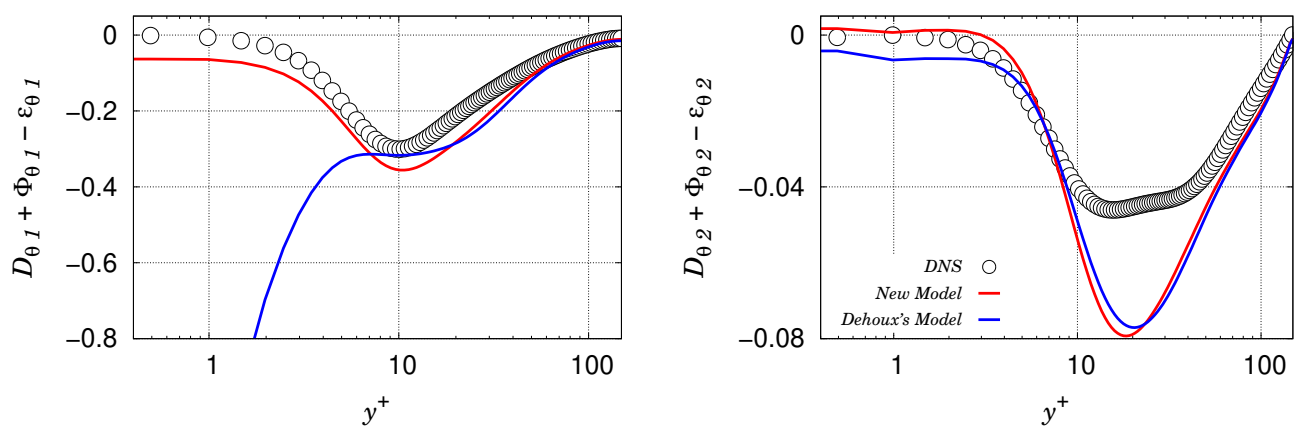

Figure 6. Sum of the molecular diffusion, the scrambling and the dissipation terms for an imposed heat flux. Left: stream-wise component; Right: wall-normal component.

terms, contrary to the very-near-wall balance (Flageul et al. 2015). The two models are asymptotically identical for this type of boundary conditions, but the coefficient $\beta$ is modeled in a different way, since Eq. (3.19) is used for the new model.

The same comparisons are performed in Figs. 6 and 7 for the cases with an imposed heat flux and with CHT, respectively. Contrary to the previous case, a superior capacity of the new model to estimate the stream-wise component of the balance can be observed. Indeed, with Dehoux's model, this component goes to infinity in both cases. This problem is mainly due to the near-wall model for $\varepsilon_{\theta 1}$ which goes to infinity at the wall as shown in Fig. 2 (left) for an imposed heat flux and in Fig. 3 (left) for CHT. In the new model, this term tends to a constant for both boundary conditions, which is the expected asymptotic behavior. Moreover, Fig. 4 (left) shows that $\phi_{\theta 1}$ is better estimated with the new model (the same profiles hold for an imposed heat flux), since it tends to zero at the wall like in the reference data.Note that although only the difference $\phi_{\theta i}-\varepsilon_{\theta i}$ appears in the transport equation for turbulent heat flux, it is important to correctly model the two terms individually, since the model for $\varepsilon_{\theta i}$ also appears in the model for $P_{\varepsilon_{\theta}}^{1}$ described in section 4.1.

Figs. 6 (right) and 7 (right) show a better estimate of the balance with the new model for the wall-normal component. Since Fig. 4 (right) shows that the two models give virtually identical results in the near-wall region for $\phi_{\theta 2}$, the improvement is only due to the better prediction of $\varepsilon_{\theta 2}$ as it can be observed in Figs. 2 (right) and 3 (right), respectively. Indeed, $\varepsilon_{\theta 2}$ tends to zero at the wall with the new model as DNS data does, 

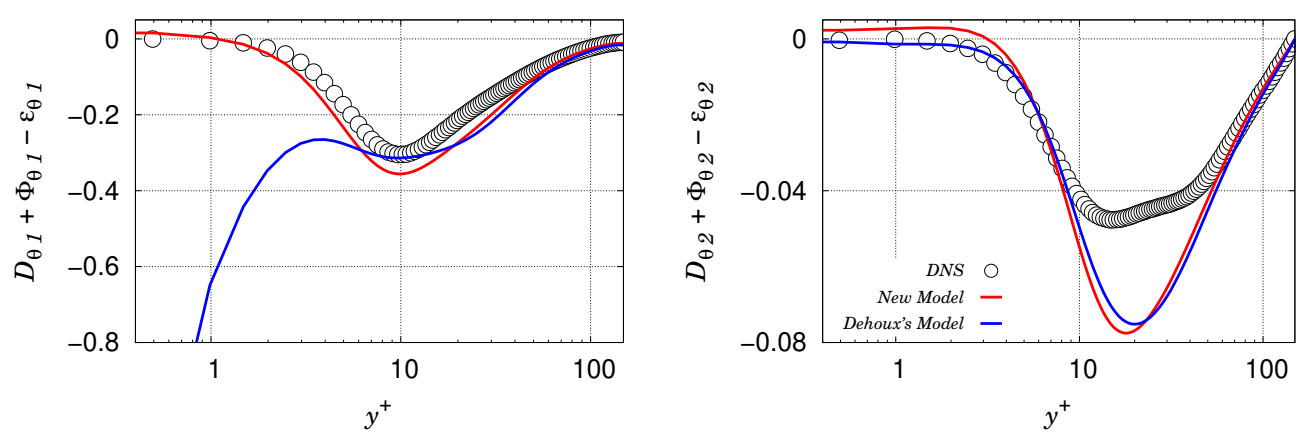

FiguRE 7. Sum of the molecular diffusion, the scrambling and the dissipation terms for Conjugate Heat Transfer (CHT). Left: stream-wise component; Right: wall-normal component.

whereas, with Dehoux's model, it goes to a constant. It is observed that the new model does not improve the results in the buffer layer: this is an intrinsic limitation of the elliptic blending approach which extends the validity of high-Reynolds number models, i.e., valid only far from the wall, to the near-wall region by satisfying the asymptotic behavior observed in the viscous sub-layer without imposing any constraint in the buffer layer.

To conclude, unlike Dehoux's model which is exclusively valid for an imposed temperature at the wall, the a priori tests confirm that the new model satisfies the asymptotic behavior of the dominant terms of near-wall budget of the turbulent heat flux. However, for this model to be complete and valid, the thermal-to-mechanical time-scale ratio $R$ must have the correct asymptotic behavior whatever the temperature boundary condition. Modeling the dissipation rate of the temperature variance $\varepsilon_{\theta}$ is then needed. The computation of $\varepsilon_{\theta}$ will also make it possible to better predict the temperature variance, without the need for an algebraic formulation of $R$.

\section{Modeling of the dissipation rate of the temperature variance}

The dissipation rate $\varepsilon_{\theta}$ is a key element in order to compute the temperature variance $\overline{\theta^{\prime 2}}$ from Eq. 2.16 and significant modeling challenges have to be tackled as its asymptotic behavior strongly depends on the thermal boundary condition. In particular, predicting $\overline{\theta^{\prime 2}}$ and $\varepsilon_{\theta}$ is crucial to obtain an accurate thermal time scale and as a consequence an accurate thermal-to-mechanical time-scale ratio $R$, which is essential in the DFM presented above, since it is involved in Eqs. (2.10), (2.11) and (3.19).

The exact transport equation for the dissipation rate of the temperature variance $\varepsilon_{\theta}=\kappa \overline{\frac{\partial \theta^{\prime}}{\partial x_{k}} \frac{\partial \theta^{\prime}}{\partial x_{k}}}$ reads

$$
\frac{D \varepsilon_{\theta}}{D t}=P_{\varepsilon_{\theta}}^{1}+P_{\varepsilon_{\theta}}^{2}+P_{\varepsilon_{\theta}}^{3}+P_{\varepsilon_{\theta}}^{4}+D_{\varepsilon_{\theta}}^{t}+D_{\varepsilon_{\theta}}^{\kappa}-Y_{\varepsilon_{\theta}}
$$

where $P_{\varepsilon_{\theta}}^{1}, P_{\varepsilon_{\theta}}^{2}, P_{\varepsilon_{\theta}}^{3}$ and $P_{\varepsilon_{\theta}}^{4}$ denote the production terms by the mean temperature gradient, the mean velocity gradient, the temperature Hessian and by turbulent interactions, respectively; $D_{\varepsilon_{\theta}}^{t}$ and $D_{\varepsilon_{\theta}}^{\kappa}$ are the turbulent and the molecular diffusion terms, and $Y_{\varepsilon_{\theta}}$ is the dissipation rate of $\varepsilon_{\theta}$. The asymptotic behavior of these terms depends on the temperature boundary conditions as shown in Table 4 . As a consequence, the asymptotic behavior of $\varepsilon_{\theta}$, and, in turn, $\overline{\theta^{\prime 2}}$, also strongly depends on the temperature boundary 


\begin{tabular}{cccccccc}
\hline & $P_{\varepsilon_{\theta}}^{1}$ & $P_{\varepsilon_{\theta}}^{2}$ & $P_{\varepsilon_{\theta}}^{3}$ & $P_{\varepsilon_{\theta}}^{4}$ & $D_{\varepsilon_{\theta}}^{t}$ & $D_{\varepsilon_{\theta}}^{\kappa}$ & $Y_{\varepsilon_{\theta}}$ \\
\hline \hline Imposed temperature & $\mathcal{O}(y)$ & $\mathcal{O}(y)$ & $\mathcal{O}(y)$ & $\mathcal{O}(y)$ & $\mathcal{O}(y)$ & $\mathcal{O}(1)$ & $\mathcal{O}(1)$ \\
\hline Imposed heat flux & $\mathcal{O}(y)$ & $\mathcal{O}(y)$ & $\mathcal{O}(y)$ & $\mathcal{O}(y)$ & $\mathcal{O}(y)$ & $\mathcal{O}(1)$ & $\mathcal{O}(1)$ \\
\hline
\end{tabular}

Conjugate Heat Transfer $(\mathrm{CHT}) \quad \mathcal{O}(1) \quad \mathcal{O}(1) \quad \mathcal{O}(y) \quad \mathcal{O}(1) \quad \mathcal{O}(y) \quad \mathcal{O}(1) \quad \mathcal{O}(1)$

TABLE 4. Asymptotic behavior of the source terms appearing in the exact transport equation for $\varepsilon_{\theta}$.
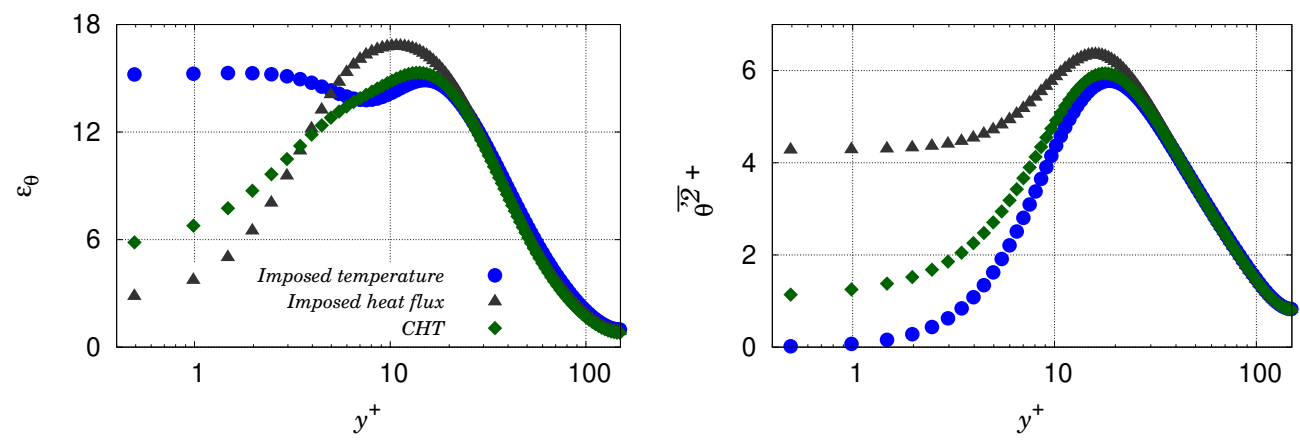

FiguRe 8 . The dissipation rate $\varepsilon_{\theta}$ (left) of the temperature variance $\overline{\theta^{\prime 2}}$ (right) from DNS Data of Flageul et al. $(2015,2017)$ for different kinds of temperature boundary conditions.

condition as illustrated by Fig. 8. Table 4 shows that $P_{\varepsilon_{\theta}}^{1}, P_{\varepsilon_{\theta}}^{2}, P_{\varepsilon_{\theta}}^{4}$ and $Y_{\varepsilon_{\theta}}$ deserve a careful attention in the near-wall region, since they are dominant in the budget, at least for the CHT case. For the two other cases, an imposed temperature and an imposed heat flux, only $Y_{\varepsilon_{\theta}}$ balances the molecular diffusion at the leading order. Note that the molecular diffusion $D_{\varepsilon_{\theta}}^{\kappa}$ naturally does not need modeling.

New models are thus derived for the four terms $P_{\varepsilon_{\theta}}^{1}, P_{\varepsilon_{\theta}}^{2}, P_{\varepsilon_{\theta}}^{4}$ and $Y_{\varepsilon_{\theta}}$. The models found in the literature for the remaining terms $P_{\varepsilon_{\theta}}^{3}$ and $D_{\varepsilon_{\theta}}^{t}$ are satisfactory, therefore they are used without modification. The production by temperature Hessian and the turbulent diffusion are modeled following Nagano (2002) and the turbulent diffusion term is modeled following Jones \& Musonge (1988). The detailed equations are given in Appendix C.

\subsection{Production terms $P_{\varepsilon_{\theta}}^{1}$ and $P_{\varepsilon_{\theta}}^{2}$}

The production term by the mean temperature gradient reads

$$
P_{\varepsilon_{\theta}}^{1}=-2 \kappa \frac{\partial \bar{\theta}}{\partial x_{j}} \overline{\frac{\partial u_{j}^{\prime}}{\partial x_{k}} \frac{\partial \theta^{\prime}}{\partial x_{k}}} .
$$



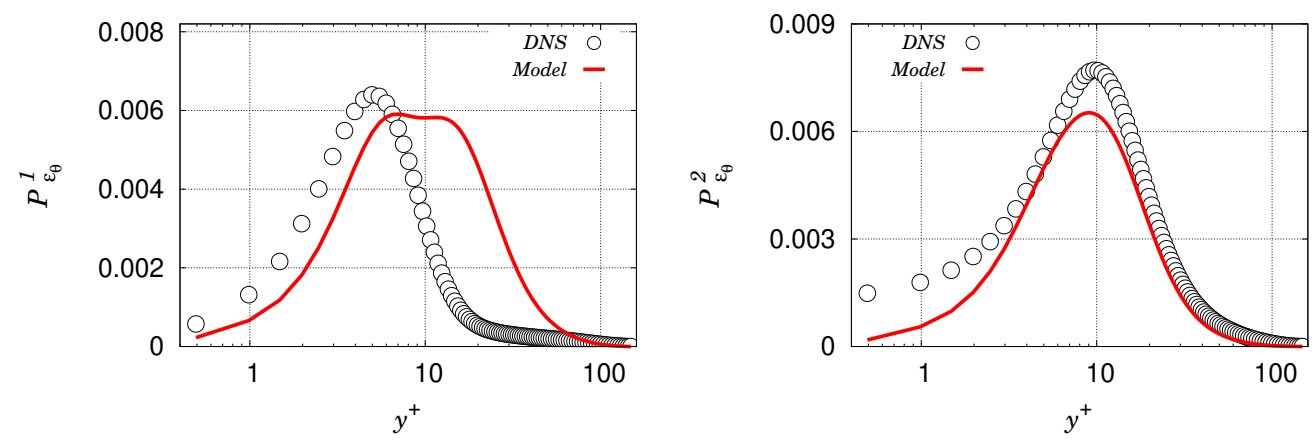

Figure 9. A priori tests: $P_{\varepsilon_{\theta}}^{1}$ (left) and $P_{\varepsilon_{\theta}}^{2}$ (right) for Conjugate Heat Transfer (CHT).

As this term is directly related to the dissipation tensor involved in the transport equation for the turbulent heat flux, $\varepsilon_{\theta j}=(\nu+\kappa) \frac{\overline{\partial u_{j}^{\prime}}}{\partial x_{k}} \frac{\partial \theta^{\prime}}{\partial x_{k}}$, it is simply modeled as

$$
P_{\varepsilon_{\theta}}^{1}=-\frac{2}{1+\operatorname{Pr}} \varepsilon_{\theta j} \frac{\partial \bar{\theta}}{\partial x_{j}},
$$

where $\varepsilon_{\theta j}=\left(1-\alpha_{\theta}\right) \varepsilon_{\theta j}^{w}$ and $\varepsilon_{\theta j}^{w}$ is given by Eq. (2.11).

The production term by the mean velocity gradient reads

$$
P_{\varepsilon_{\theta}}^{2}=-2 \kappa \frac{\partial \overline{u_{j}}}{\partial x_{k}} \frac{\overline{\partial \theta^{\prime}}}{\partial x_{k}} \frac{\partial \theta^{\prime}}{\partial x_{j}}
$$

The tensor $\varepsilon_{i j}^{\theta}=2 \kappa \overline{\frac{\partial \theta^{\prime}}{\partial x_{i}} \frac{\partial \theta^{\prime}}{\partial x_{j}}}$ is very similar to the dissipation tensor $\varepsilon_{i j}$ in the Reynoldsstress tensor equation, and, in particular, is linked to the smallest scales of the turbulent thermal field. Therefore, the same modeling strategy as for $\varepsilon_{i j}$ is adopted: it is assumed that $\varepsilon_{i j}^{\theta}$ is isotropic far from the wall and exhibits the same anisotropy as the Reynoldsstress tensor in the near-wall region, such that it is formulated as

$$
\varepsilon_{i j}^{\theta}=\left(1-\alpha_{\theta}\right) \varepsilon_{i j}^{\theta w}+\alpha_{\theta} \varepsilon_{i j}^{\theta h},
$$

where

$$
\varepsilon_{i j}^{\theta h}=\frac{2}{3} \varepsilon_{\theta} \delta_{i j} \quad \text { and } \quad \varepsilon_{i j}^{\theta w}=\frac{\overline{u_{i}^{\prime} u_{j}^{\prime}}}{k} \varepsilon_{\theta} .
$$

Hence, the production by the mean velocity gradient is modeled as

$$
P_{\varepsilon_{\theta}}^{2}=-c_{\varepsilon_{\theta 1}} \varepsilon_{j k}^{\theta} \frac{\partial \overline{u_{j}}}{\partial x_{k}} .
$$

For the sake of concision, the a priori estimates of the two production terms are only shown for CHT in Fig. 9. Conclusions are similar for the two other boundary conditions. For $P_{\varepsilon_{\theta}}^{1}$, the near-wall behavior of DNS is correctly recovered. A relatively weak prediction in the buffer layer is observed (for all the boundary conditions) which is due to an overestimated dissipation rate $\varepsilon_{\theta 2}$ in this region. For $P_{\varepsilon_{\theta}}^{2}$, the predictions are satisfactory far from the wall. The modeled production goes to zero at the wall whatever the thermal boundary conditions, which is not the case according to DNS data for CHT. However, in the latter case, the production by the mean velocity gradient tends towards a small non-zero value at the wall which makes the model acceptable. 

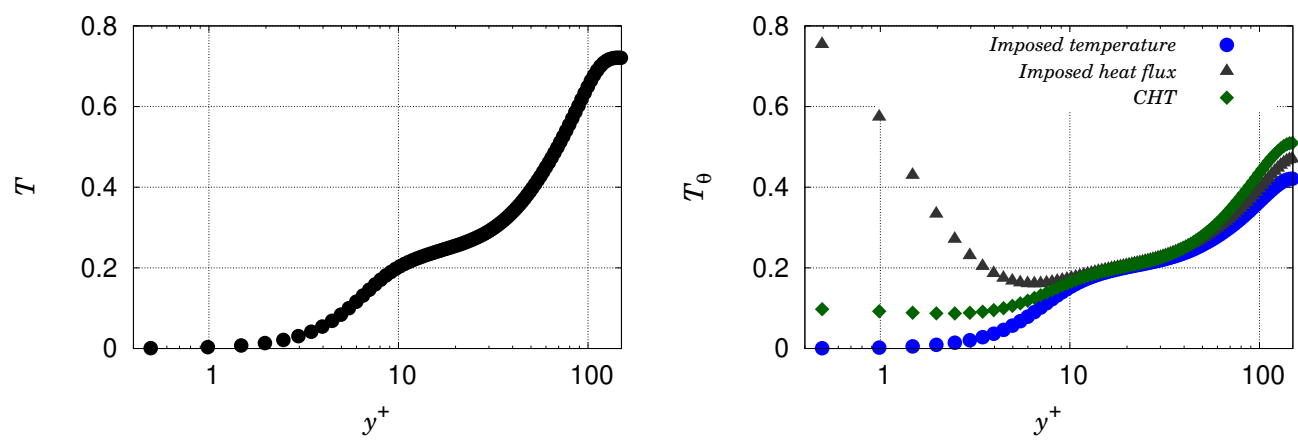

Figure 10. The dynamic time scale ratio $\mathcal{T}$ (left) and the thermal time-scale ratio $\mathcal{T}_{\theta}$ (right) from DNS Data of Flageul et al. $(2015,2017)$ for different kinds of temperature boundary conditions.

\subsection{Difference between the turbulent production and the dissipation $P_{\varepsilon_{\theta}}^{4}-Y_{\varepsilon_{\theta}}$}

The difference between the turbulent production, $P_{\varepsilon_{\theta}}^{4}$, and the dissipation rate of $\varepsilon_{\theta}$, $Y_{\varepsilon_{\theta}}$, reads

$$
P_{\varepsilon_{\theta}}^{4}-Y_{\varepsilon_{\theta}}=-2 \kappa \overline{\frac{\partial \theta^{\prime}}{\partial x_{j}} \frac{\partial \theta^{\prime}}{\partial x_{k}} \frac{\partial u_{j}^{\prime}}{\partial x_{k}}}-2 \kappa^{2} \overline{\frac{\partial^{2} \theta^{\prime}}{\partial x_{j} \partial x_{k}} \frac{\partial^{2} \theta^{\prime}}{\partial x_{j} \partial x_{k}}} .
$$

Newman et al. (1981) showed that these two terms are dominant in the absence of walls. In particular, for homogeneous turbulence without mean velocity and temperature gradients, these two terms are the only non-zero terms and drive the time evolution of the fluctuating thermal field. Since there is no theoretical case in which these two terms can be distinguished, Jones \& Musonge (1988) modeled these two terms as a whole

$$
P_{\varepsilon_{\theta}}^{4}-Y_{\varepsilon_{\theta}}=c_{\varepsilon_{\theta}}^{h 1} \frac{\varepsilon_{\theta}}{k} P_{k}+c_{\varepsilon_{\theta}}^{h 2} \frac{\varepsilon}{k} P_{\theta}-c_{\varepsilon_{\theta 3}} \frac{\varepsilon_{\theta}}{\mathcal{T}_{\theta}}-c_{\varepsilon_{\theta 4}} \frac{\varepsilon_{\theta}}{\mathcal{T}} .
$$

In order to extend the validity of this model down to the wall, the elliptic bending approach is used

$$
P_{\varepsilon_{\theta}}^{4}-Y_{\varepsilon_{\theta}}=\left(1-\alpha_{\theta}\right)\left(P_{\varepsilon_{\theta}}^{w}-Y_{\varepsilon_{\theta}}^{w}\right)+\alpha_{\theta}\left(P_{\varepsilon_{\theta}}^{h}-Y_{\varepsilon_{\theta}}^{h}\right)
$$

Jones \& Musonge (1988) have introduced the term $c_{\varepsilon_{\theta 4}} \varepsilon_{\theta} / \mathcal{T}$ so that the thermal-tomechanical time-scale ratio $R$ is constant in the case of heated grid turbulence. It is preferable not to introduce this term, so that in this case $R$ is not constant but tends asymptotically towards an equilibrium value, close to 0.5 . The quasi-homogeneous model is therefore written as follows

$$
P_{\varepsilon_{\theta}}^{h}-Y_{\varepsilon_{\theta}}^{h}=c_{\varepsilon_{\theta}}^{h 1} \frac{\varepsilon_{\theta}}{k} P_{k}+c_{\varepsilon_{\theta}}^{h 2} \frac{\varepsilon}{k} P_{\theta}-c_{H} \frac{\varepsilon_{\theta}}{\tau_{\theta}} .
$$

where

$$
\tau_{\theta}=\max \left(\mathcal{T}_{\theta}, C_{T_{\theta}} \sqrt{\frac{\nu}{\varepsilon}} \frac{1}{P r}\right) .
$$

Regarding the near-wall models $P_{\varepsilon_{\theta}}^{w}$ and $Y_{\varepsilon_{\theta}}^{w}$, they have to reproduce the asymptotic behavior of $P_{\varepsilon_{\theta}}^{4}$ and $Y_{\varepsilon_{\theta}}$ at the wall. The DNS data from Flageul et al. $(2015,2017)$ show that the asymptotic behavior of $P_{\varepsilon_{\theta}}^{4}$ is virtually unaffected by the thermal boundary condition, as shown in Fig. 11 (left). The near-wall part $P_{\varepsilon_{\theta}}^{w}$ is modeled using the ratio of the thermal production $P_{\theta}$ and the mixed time-scale $\sqrt{\mathcal{T} \mathcal{T}_{\theta}}$, which is barely affected 

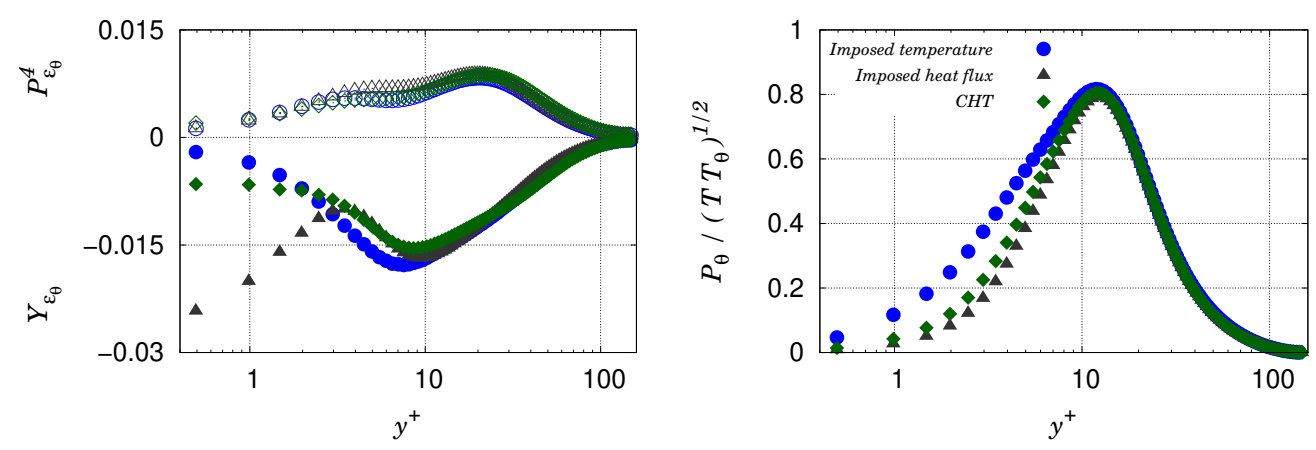

FIgURE 11. The turbulent production $P_{\varepsilon_{\theta}}^{4}$ and the dissipation rate $Y_{\varepsilon_{\theta}}$ (left) and the thermal production $P_{\theta}$ divided by the mixed time-scale $\sqrt{\mathcal{T} \mathcal{T}_{\theta}}$ from the DNS Data of Flageul et al. (2015, 2017) for different kinds of temperature boundary conditions.
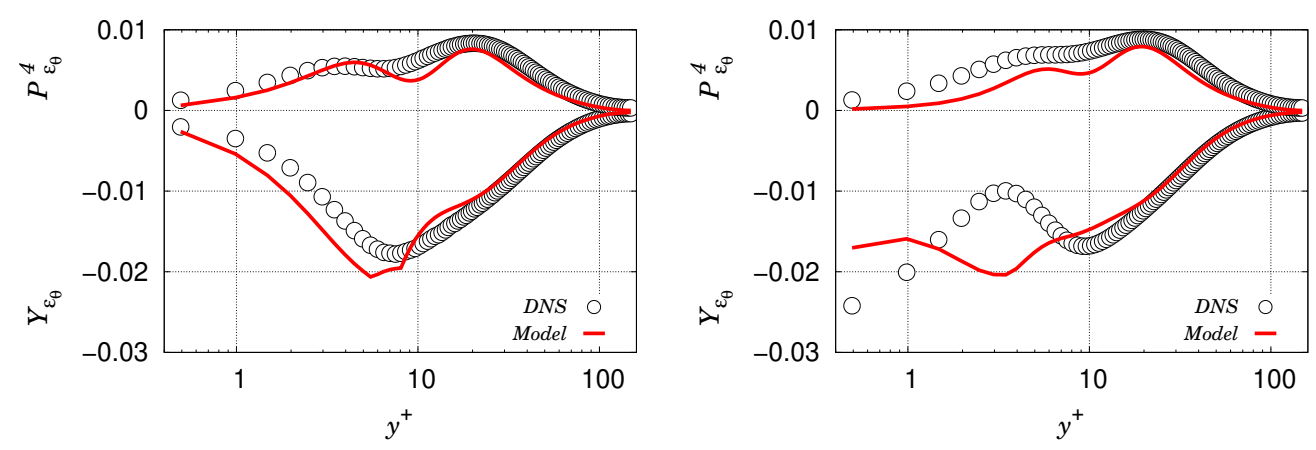

Figure 12. A priori tests: $P_{\varepsilon_{\theta}}^{4}$ and $Y_{\varepsilon_{\theta}}$ for an imposed temperature at the wall (left) and for an imposed heat flux (right).

by the thermal boundary condition, Fig. 11 (right):

$$
P_{\varepsilon_{\theta}}^{w}=c_{\varepsilon_{\theta}}^{w 1}\left[1-c_{\varepsilon_{\theta}}^{w 2}\left(1-\alpha_{\theta}\right) \frac{P_{k}}{\varepsilon}\right] \frac{P_{\theta}}{\varepsilon_{\theta}} \frac{\varepsilon_{\theta}}{\sqrt{\mathcal{T} \mathcal{T}_{\theta}}} .
$$

The term in square brackets is introduced to improve the predictions in the buffer layer, in a way similar to Eqs. (2.10) and (2.11) in Dehoux's model. Table 5 gives the asymptotic behavior of $P_{\theta}, \sqrt{\mathcal{T} \mathcal{T}_{\theta}}$ and $P_{\varepsilon_{\theta}}^{4}$ for the different thermal boundary conditions. The right asymptotic behavior is obtained for $P_{\varepsilon_{\theta}}^{w}$ and, consequently, for $P_{\varepsilon_{\theta}}^{4}$, in the cases of an imposed temperature and CHT. For an imposed heat flux, $P_{\varepsilon_{\theta}}^{w}$ goes to a non-zero value at the wall while $P_{\varepsilon_{\theta}}^{4}$ tends to zero, as shown in Tab. 4. However, the a priori tests shown in Fig. 12 (right) indicate that the limiting value is very small and the model gives satisfactory results when compared to the DNS. As a consequence, the model $P_{\varepsilon_{\theta}}^{w}$ given by Eq. (4.13) is also used in the case of an imposed heat flux. The a priori tests show that the present model for $P_{\varepsilon_{\theta}}^{4}$ correctly reproduces the near and far-from-the-wall regions for all the thermal boundary conditions, as illustrated by Figs. 12 and 13 .

With regard to $Y_{\varepsilon_{\theta}}$, its asymptotic behavior strongly depends on the thermal boundary condition at the wall, as seen in Fig. 11. Indeed, $Y_{\varepsilon_{\theta}}$ tends to radically different non-zero values at the wall: for an imposed temperature, the value is very small; for an imposed heat flux, $Y_{\varepsilon_{\theta}}^{w}$ reaches its maximum at the wall and exhibits a secondary peak in the buffer 


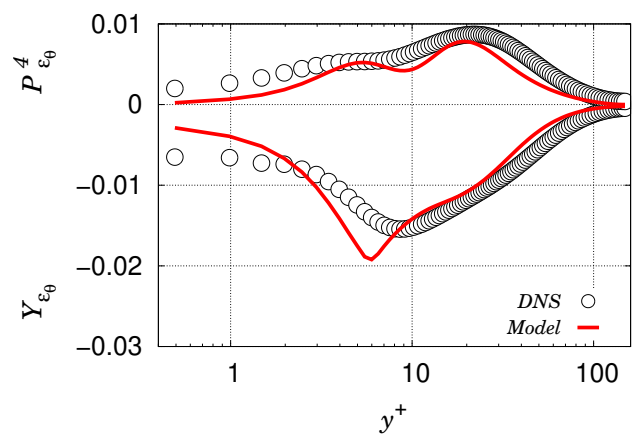

Figure 13. A priori tests: $P_{\varepsilon_{\theta}}^{4}$ and $Y_{\varepsilon_{\theta}}$ for Conjugate Heat Transfer (CHT).

\begin{tabular}{cccc}
\hline & $P_{\theta}$ & $\sqrt{\mathcal{T} \mathcal{T}_{\theta}}$ & $P_{\varepsilon_{\theta}}^{w}$ \\
\hline \hline Imposed temperature & $\mathcal{O}\left(y^{3}\right)$ & $\mathcal{O}\left(y^{2}\right)$ & $\mathcal{O}(y)$ \\
\hline Imposed heat flux & $a_{1} t_{0} \frac{\partial T_{0}}{\partial x} y+\mathcal{O}\left(y^{2}\right)$ & $\mathcal{O}(y)$ & $\mathcal{O}(1)$ \\
\hline Conjugate Heat Transfer (CHT) & $a_{1} t_{0} \frac{\partial T_{0}}{\partial x} y+\mathcal{O}\left(y^{2}\right)$ & $\mathcal{O}(y)$ & $\mathcal{O}(1)$ \\
\hline
\end{tabular}

TABle 5. Asymptotic behavior of the thermal production $P_{\theta}$, the mixed time-scale $\sqrt{T T_{\theta}}$ and the near-wall model $P_{\varepsilon_{\theta}}^{w}$ for all types of temperature boundary conditions.

layer; for CHT, an intermediate value is reached. Reproducing this particular behavior is crucial, since $Y_{\varepsilon_{\theta}}$ is dominant in the budget of $\varepsilon_{\theta}$, and is very challenging.

To this end, two key parameters are used to sensitize the model to the thermal boundary condition,

$$
\sqrt{\frac{P r}{R}} \quad \text { with } \quad R=\frac{\mathcal{T}}{\mathcal{T}_{\theta}}
$$

and

$$
\frac{\sigma_{\theta}}{\varepsilon_{\theta}} \quad \text { with } \quad \sigma_{\theta}=\kappa\left(\frac{\partial \sqrt{\overline{\theta^{\prime 2}}}}{\partial y}\right)^{2} .
$$

The first parameter $\sqrt{\operatorname{Pr}} / \sqrt{R}$, which was already introduced in the near-wall models $\phi_{\theta i}^{w}$ and $\varepsilon_{\theta i}^{w}$ presented in section 3 , distinguishes an imposed wall temperature from the other two cases, as illustrated by Fig. 14 (left). However, in order to reproduce the particular behaviour of $Y_{\varepsilon_{\theta}}$ described above, a distinction must be made between the case of an imposed heat flux case and the case of CHT, hence the introduction of the 

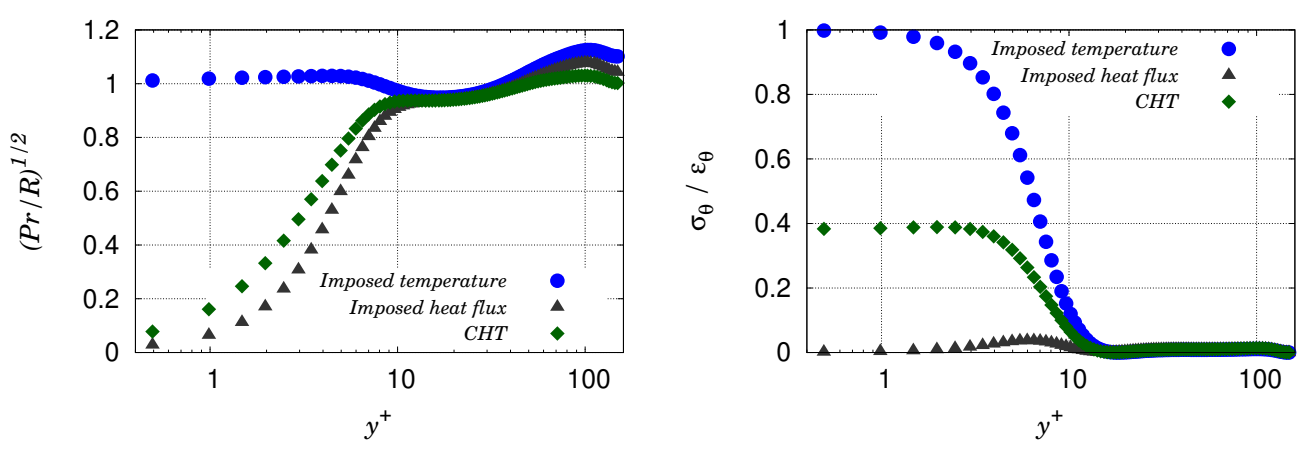

Figure 14. The two parameters $\sqrt{\operatorname{Pr} / R}$ (left) and $\sigma_{\theta} / \varepsilon_{\theta}$ used to distinguish between the different kinds of temperature boundary condition, from DNS Data of Flageul et al. (2015, 2017).

second parameter $\sigma_{\theta} / \varepsilon_{\theta}$. This parameter proposed by Yang et al. (2019) goes to very different values at the wall in the three cases, as shown in Fig. 14 (right).

Combining these two key parameters, the following expression for the near-wall model $Y_{\varepsilon_{\theta}}^{w}$ is proposed

$$
\begin{gathered}
Y_{\varepsilon_{\theta}}^{w}=\left[\left(1-\sqrt{\frac{P r}{R}}\right)\left(1-c_{w}\left(1-\alpha_{\theta}\right) \frac{P_{\theta}}{\varepsilon_{\theta}}\right)\left(c_{N}-\frac{\sigma_{\theta}}{\varepsilon_{\theta}} c_{C}\right)\right. \\
\left.+c_{D} \sqrt{\frac{P r}{R}}\right] \frac{\varepsilon_{\theta}}{\sqrt{\tau_{\varepsilon_{\theta}} \tau_{\theta}}} \sqrt{\frac{\mathcal{T}_{\theta}}{\tau_{\theta}}}
\end{gathered}
$$

where

$$
\tau_{\varepsilon_{\theta}}=\max \left(\mathcal{T}, C_{\mathcal{T}} \sqrt{\frac{\nu}{\varepsilon}}\right)
$$

is similar to Durbin's mechanical time scale (2.5), but with the variable coefficient

$$
C_{\mathcal{T}}=\left(1-\sqrt{\frac{P r}{R}}\right)\left(c_{T_{N}}+\frac{\sigma_{\theta}}{\varepsilon_{\theta}} c_{T_{C}}\right)+c_{T_{D}} \sqrt{\frac{P r}{R}} .
$$

As illustrated by Fig. 15, the purpose of this expression for $C_{\mathcal{T}}$ is to impose different values of the coefficient for the different thermal boundary conditions, and the constants $c_{T_{D}}, c_{T_{N}}$, and $c_{T_{C}}$, where $D, N$ and $C$ stand for Dirichlet, Neuman and Conjugate, respectively, are calibrated in order to reproduce at best the behavior of $Y_{\varepsilon_{\theta}}$ in the nearwall region. Indeed, since $\mathcal{T}$ goes to zero at the wall regardless of the thermal boundary condition, the wall-limiting value of $\tau_{\varepsilon_{\theta}}$ is given by the second term in Eq. (4.17), and modifying the coefficient $C_{\mathcal{T}}$ makes it possible to ajust the near-wall behavior of $Y_{\varepsilon_{\theta}}^{w}$. The particular values reached at the wall by the two parameters $\sqrt{\operatorname{Pr} / R}$ and $\sigma_{\theta} / \varepsilon_{\theta}$ are such that the coefficients $c_{T_{D}}, c_{T_{N}}$, and $c_{T_{C}}$ are active for an imposed temperature, an imposed heat flux and CHT, respectively.

At the wall, $\tau_{\varepsilon_{\theta}}, \tau_{\theta}$ and $\varepsilon_{\theta}$ always tend to non-zero values. Therefore, the asymptotic behavior of $Y_{\varepsilon_{\theta}}^{w}$ is driven by $\sqrt{\mathcal{T}_{\theta}}$. As a consequence, the modeled $Y_{\varepsilon_{\theta}}^{w}$ correctly goes to a non-zero value at the wall for an imposed heat flux and CHT, but not for an imposed temperature, as summarized in Tab. 6. However, since Fig. 11 (left) shows that, for an 
Imposed temperature Imposed heat flux Conjugate Heat Transfer (CHT)

\begin{tabular}{cccc}
\hline \hline Modeled $Y_{\varepsilon_{\theta}}^{w}$ & $\mathcal{O}(y)$ & $\mathcal{O}(1)$ & $\mathcal{O}(1)$ \\
Exact $Y_{\varepsilon_{\theta}}$ & $\mathcal{O}(1)$ & $\mathcal{O}(1)$ & $\mathcal{O}(1)$ \\
\hline
\end{tabular}

TABLE 6. Asymptotic behavior of the near-wall model for $Y_{\varepsilon_{\theta}}^{w}$ and the exact $Y_{\varepsilon_{\theta}}$.

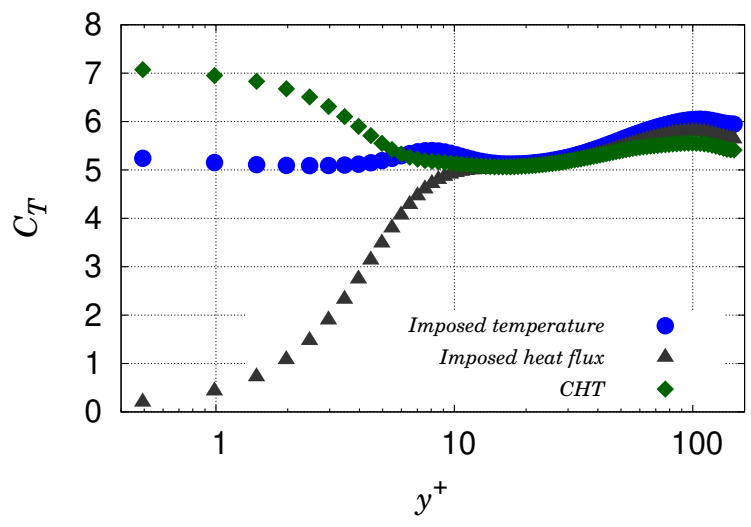

FiguRE 15. $C_{\mathcal{T}}$ coefficient profile for different temperature boundary conditions from DNS Data of Flageul et al. $(2015,2017)$.

imposed temperature, the exact $Y_{\varepsilon_{\theta}}$ goes to a very small non-zero value, the fact that the modeled $Y_{\varepsilon_{\theta}}^{w}$ tends to zero is acceptable.

A priori tests are shown in Figs. 12 and 13. These tests confirm that, in the case of an imposed temperature, although the wall asymptotic behavior in $\mathcal{O}(1)$ is not reproduced by the model, the profile of $Y_{\varepsilon_{\theta}}$ is correctly predicted. In the two other cases, the values at the wall are underestimated, but it is to be noted that the coefficient $C_{\mathcal{T}}$ is calibrated based on the full computations shown in the next section, not on the a priori tests. One can observe slope discontinuities for the modeled $Y_{\varepsilon_{\theta}}$ that are due to the max functions used in the time-scales $\tau_{\theta}$ and $\tau_{\varepsilon_{\theta}}$. Far from the wall, the model tends to the quasi-homogeneous model, which is close to the DNS data for all the thermal boundary conditions. The full set of equations and coefficients is available in Appendix C.

\section{Model validation based on full computations}

Full computations are carried out with EDF in-house open-source finite volumes CFD solver Code_Saturne†. Details about the finite volume discretization scheme can be found in Archambeau et al. (2004). 


\subsection{Governing equations}

The channel flow configurations in the forced convection regime of Flageul et al. (2015) are used for validation. The friction Reynolds number $R e_{\tau}$ and the Prandtl number $\mathrm{Pr}$ are equal to 149 and 0.71 , respectively. The non-dimensional kinematic viscosity and thermal diffusivity are then equal to $1 / R e_{\tau}$ and $1 /\left(R e_{\tau} P r\right)$, respectively.

In addition to standard mean velocity transport equations driven by a pressure gradient, the following equation is solved for the mean temperature (Kasagi et al. 1992)

$$
\frac{\partial \bar{\theta}}{\partial t}+\bar{u}_{j} \frac{\partial \bar{\theta}}{\partial x_{j}}=\frac{1}{R e_{\tau} \operatorname{Pr}} \frac{\partial^{2} \bar{\theta}}{\partial x_{j} \partial x_{j}}-\frac{\partial \overline{u_{j}^{\prime} \theta^{\prime}}}{\partial x_{j}}+\frac{\bar{u}_{1}}{u_{b}}
$$

where $u_{b}$ the bulk velocity. In order to impose periodicity in the stream-wise direction, the mean temperature is decomposed into a periodic and a linearly variable part. As a consequence, the source term $\frac{\bar{u}_{1}}{u_{b}}$ on the r.h.s. of the mean temperature equation $(5.1)$.

Periodic boundary conditions are imposed in the stream-wise $(x)$ direction and symmetries in the span-wise $(z)$ direction and at the central plane $(y=\delta)$, for all the variables. At the wall $(y=0)$, standard no-slip boundary conditions are imposed for the mean velocity and the turbulent variables.

Three types of thermal wall boundary conditions are imposed: (i) an imposed wall temperature with $\bar{\theta}=0, \overline{\theta^{\prime 2}}=0$ and $\varepsilon_{\theta}=\lim _{y \rightarrow 0}\left(\kappa \overline{\theta^{\prime 2}} / y^{2}\right)$; (ii) an imposed heat flux, which reads $\partial \bar{\theta} / \partial y=-\operatorname{Pr} \operatorname{Re}_{\tau}, \frac{\partial \overline{\theta^{\prime 2}}}{\partial y}=0$ and $\frac{\partial \varepsilon_{\theta}}{\partial y}=0$; (iii) CHT for which a solid part having a thickness equal to the channel half-width is added, and the heat flux $\partial \bar{\theta} / \partial y=-\operatorname{Pr} R e_{\tau}$ is imposed at the outer boundary of the solid part.

In the latter case, the solid has the same properties as the fluid; in addition to the mean temperature, its conductive flux and its variance, the dissipation of the variance is continuous at the fluid-solid interface. The equations resolved in the solid part read

$$
\begin{gathered}
\frac{\partial \bar{\theta}}{\partial t}=\frac{\partial}{\partial x_{j}}\left(\kappa_{s} \frac{\partial \bar{\theta}}{\partial x_{j}}\right), \\
\frac{D \overline{\theta^{\prime 2}}}{D t}=\frac{\partial}{\partial x_{j}}\left(\kappa_{s} \frac{\partial \overline{\theta^{\prime 2}}}{\partial x_{j}}\right)-2 \varepsilon_{\theta}, \\
\frac{\partial \varepsilon_{\theta}}{\partial t}=\frac{\partial}{\partial x_{j}}\left(\kappa_{s} \frac{\partial \varepsilon_{\theta}}{\partial x_{j}}\right)-c_{\varepsilon_{\theta_{s}}} \frac{\varepsilon_{\theta}}{\mathcal{T}_{\theta}},
\end{gathered}
$$

where $\kappa_{s}=\frac{1}{R e_{\tau} \operatorname{Pr}}$ stands for the non-dimensional thermal diffusivity of the solid. $\varepsilon_{\theta}=$ $\kappa_{s} \frac{\overline{\partial \theta^{\prime}}}{\partial x_{k}} \frac{\partial \theta^{\prime}}{\partial x_{k}}$ is the dissipation of the variance. In Eq. (5.4), the dissipation term is modeled in a similar way to the homogeneous model in the fluid domain, using $c_{\varepsilon_{\theta_{s}}} \frac{\varepsilon_{\theta}}{\mathcal{T}_{\theta}}$. The constant $c_{\varepsilon_{\theta_{s}}}$ has been estimated a priori using the DNS data from Flageul et al. (2015) and is taken equal to 3 .

All the results are plotted in wall units. Since the mean velocity profile is not affected by the thermal boundary condition, it is not shown here for the sake of concision. The interested reader is invited to refer to Manceau (2015). Fig. 16 shows the profiles of the non-dimensional temperature $\bar{\theta}^{+}=\left(\bar{\theta}-\bar{\theta}_{w}\right) / T_{\tau}$. As a consequence of the accurate prediction of the wall-normal turbulent heat flux, as will be shown below, the temperature 

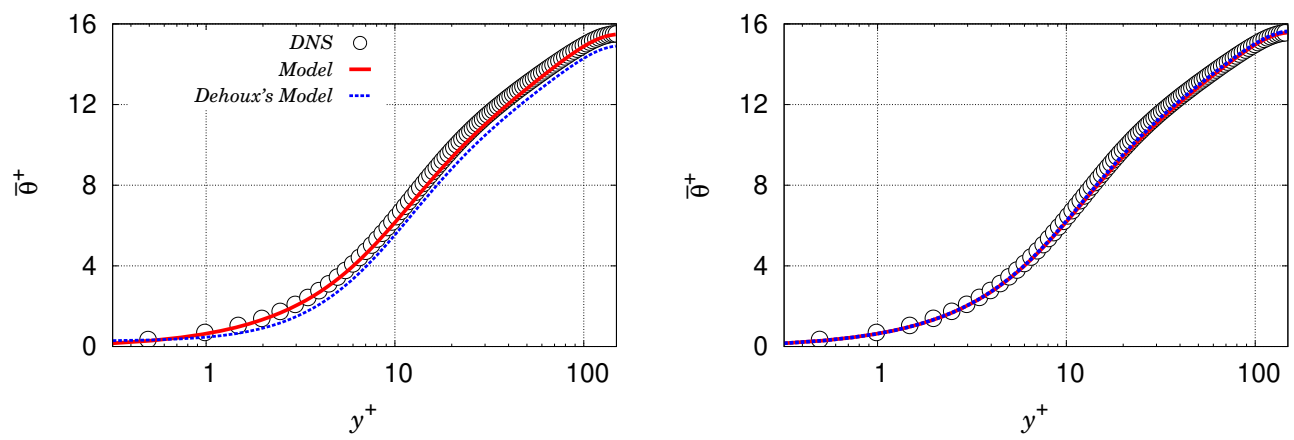

Figure 16. Predicted mean temperature with an imposed heat flux (left) and Conjugate Heat Transfer (CHT) (right) at the wall.

profile is correctly reproduced with the new model. Dehoux's model also gives correct results for CHT, but the mean temperature predicted by this model is less accurate than with the new model for an imposed heat flux.

\subsection{The temperature variance $\overline{\theta^{\prime 2}}$ and its dissipation rate $\varepsilon_{\theta}$}

Figs. 17, 18 and 19 compare the temperature variance (left) and its dissipation rate (right) obtained with the new model to the DNS data from Flageul et al. (2015) and to the results obtained with Dehoux's model. The prediction of these two quantities are clearly improved with the new model even in the case of an imposed temperature, for which Dehoux's model is asymptotically correct. Dehoux's model is virtually insensitive to the thermal boundary condition, as $\varepsilon_{\theta}$ always goes to the same large value at the wall, such that the temperature variance tends to zero in all the cases. In addition to sensitizing the model to the thermal boundary condition, a favorable side effect of solving the $\varepsilon_{\theta}$-equation is a significant improvement of the predictions of $\varepsilon_{\theta}$ and $\overline{\theta^{\prime 2}}$ far from the wall.

Figs. 20, 21 and 22 compare the budgets of $\overline{{\theta^{\prime}}^{2}}$ and $\varepsilon_{\theta}$ with the DNS data of Flageul et al. (2015) for an imposed temperature, an imposed heat flux and CHT, respectively. Although the prediction of the different terms of the budget of $\varepsilon_{\theta}$ is not perfect, these figures globally suggest that the satisfactory prediction of $\overline{\theta^{\prime 2}}$ and $\varepsilon_{\theta}$ shown above is due to a correct reproduction of all the physical mechanisms playing a role in the dynamics of these variables. The left parts of these figures show an excellent prediction of the budget of the temperature variance for all the thermal boundary conditions, which is due to the good prediction of $\varepsilon_{\theta}$ using the new modeled equation, but also to the good prediction of the turbulent heat flux that enters production, as will be shown in section 5.3.

In particular, these computations confirm that the new model for $Y_{\varepsilon_{\theta}}$ is successful in reproducing the dramatic modification of the near-wall behavior of this quantity when boundary conditions vary. This is the cornerstone of the present DFM, since this term is always a leading-order term, as shown in Table 4.

Finally, Fig. 23 shows the temperature variance and its dissipation rate in the solid part in the case of CHT. $G=1$ and $G_{2}=1$ are the fluid-to-solid ratios of the thermal diffusivity and the thermal conductivity, respectively. A virtually perfect agreement with the DNS is obtained for $\overline{\theta^{\prime 2}}$ and $\varepsilon_{\theta}$. Since these two quantities are continuous across the interface, this good prediction is linked to the performance of the model on the fluid side. Moreover, the choice of the constant $c_{\varepsilon_{\theta_{s}}}$ in Eq. 5.4 appears adequate as it drives the 

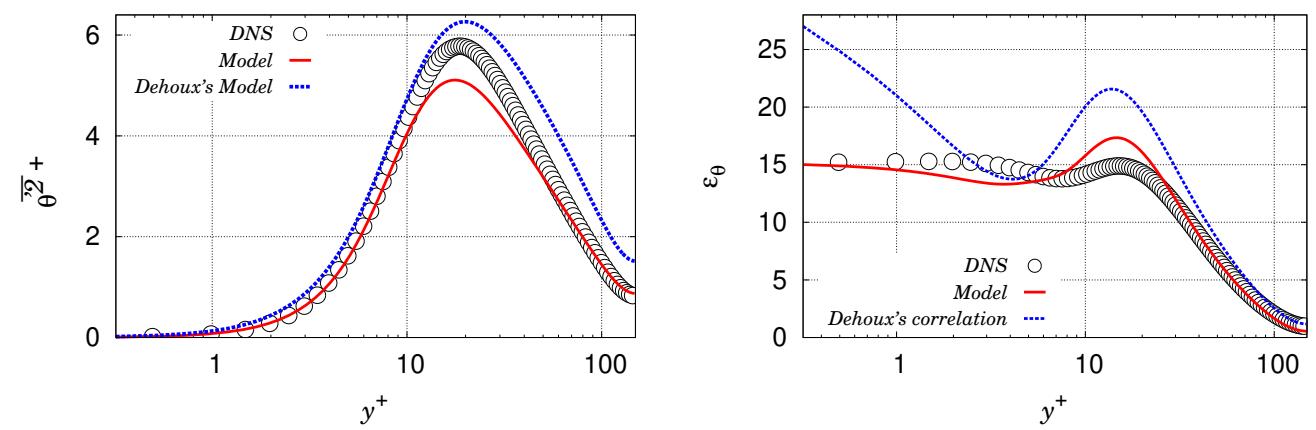

Figure 17. A posteriori temperature variance $\overline{\theta^{\prime 2}}$ (left) and its dissipation $\varepsilon_{\theta}$ (right) for an imposed temperature at the wall.
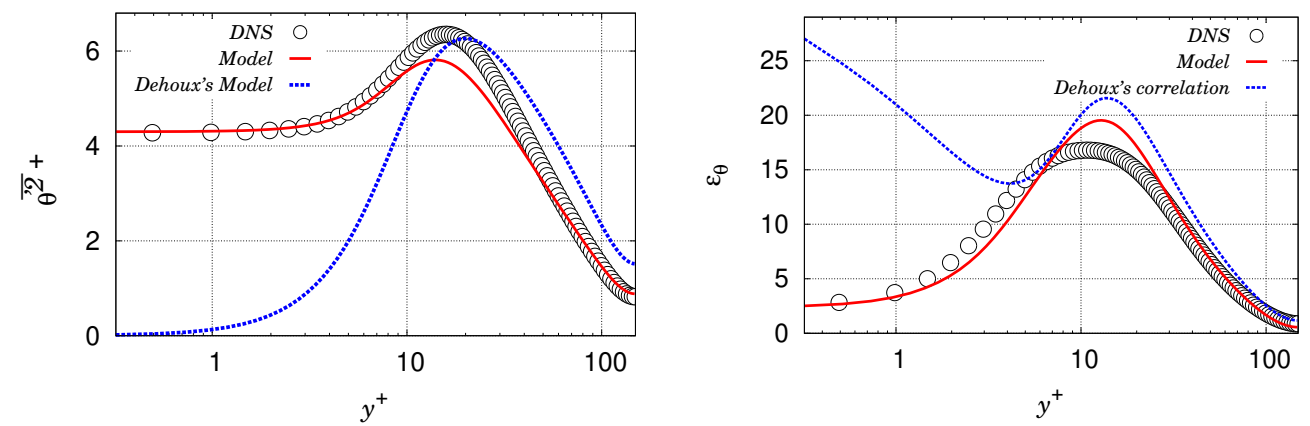

Figure 18. A posteriori temperature variance $\overline{\theta^{\prime 2}}$ (left) and its dissipation $\varepsilon_{\theta}$ (right) for an imposed heat flux at the wall.
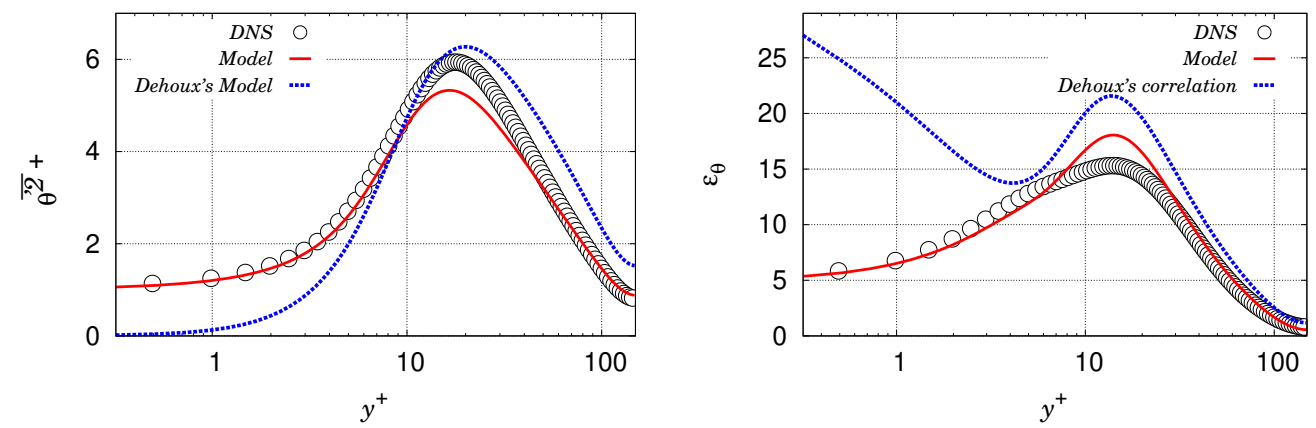

Figure 19. A posteriori temperature variance $\overline{\theta^{\prime 2}}$ (left) and its dissipation $\varepsilon_{\theta}$ (right) for the Conjugate Heat Transfer (CHT) case.

decrease with the distance to the interface of the dissipation in the solid, which in turn leads to the decrease of the temperature variance.

\subsection{Turbulent heat flux}

In Fig. 24, in order to confirm the favorable results obtained by a priori tests in section 3.3 , the near-wall balance of transport equation for the turbulent heat flux, $D_{\theta i}^{\nu+\kappa}+\phi_{\theta i}-$ $\varepsilon_{\theta i}$, i.e., the sum of the molecular diffusion, the scrambling and the dissipation terms, 

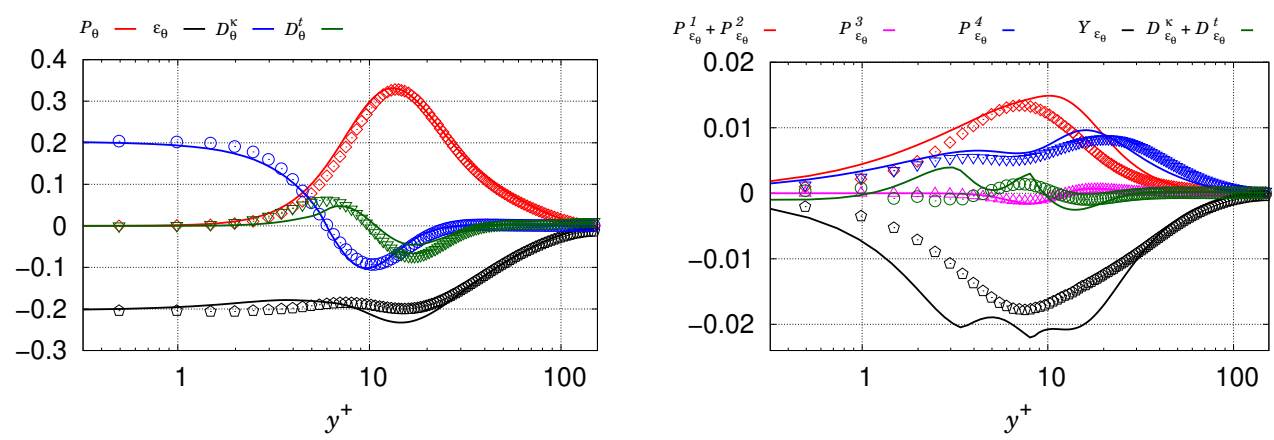

Figure 20. Temperature variance $\overline{\theta^{\prime 2}}$ budgets (left) and the dissipation rate $\varepsilon_{\theta}$ budgets (right) for an imposed temperature at the wall. The symbols stand for DNS data and the solid lines represent the present model.
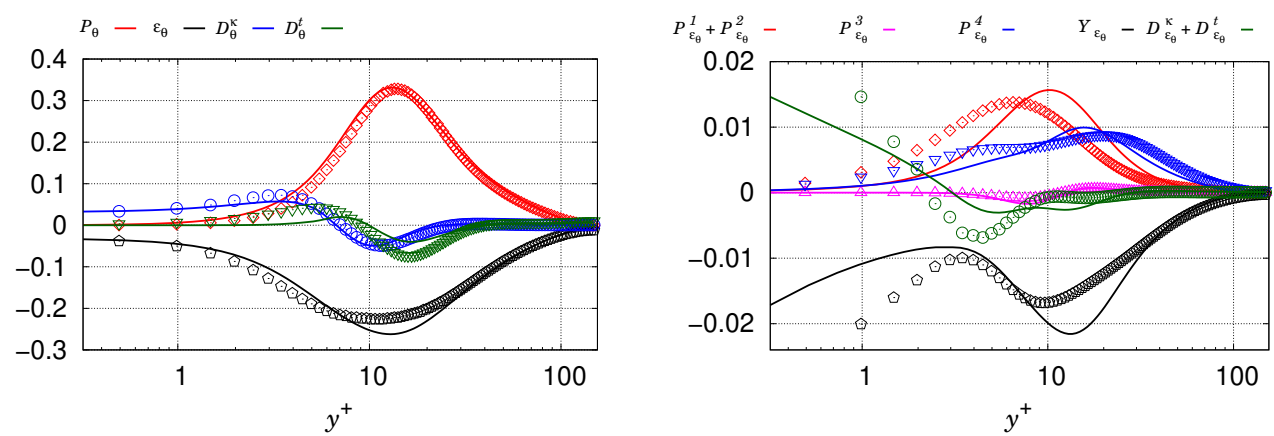

Figure 21. Temperature variance $\overline{\theta^{\prime 2}}$ budgets (left) and the dissipation rate $\varepsilon_{\theta}$ budgets (right) for an imposed heat flux. The symbols stand for DNS data and the solid lines represent the present model.
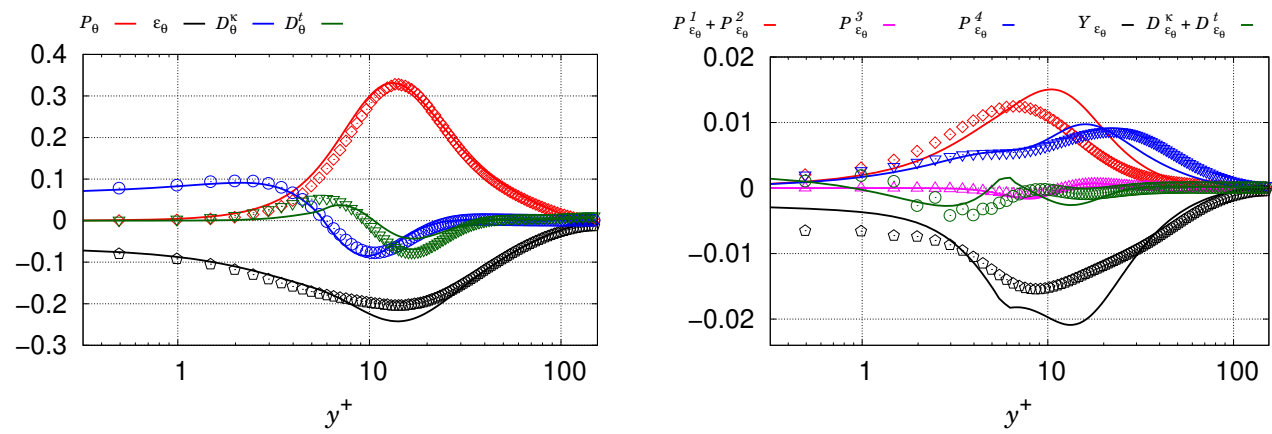

Figure 22. Temperature variance $\overline{\theta^{\prime 2}}$ budgets (left) and the dissipation rate $\varepsilon_{\theta}$ budgets (right) for Conjugate Heat Transfer (CHT). The symbols stand for DNS data and the solid lines represent the present model.

is compared with the DNS data of Flageul et al. (2015) for the CHT case. As expected from the asymptotic analysis shown in section 3.2 and the a priori tests, the new model accurately reproduces the near-wall behavior of these terms, which remain dominant up to $y^{+} \simeq 5$. These results form a solid basis for the correct reproduction of the turbulent 

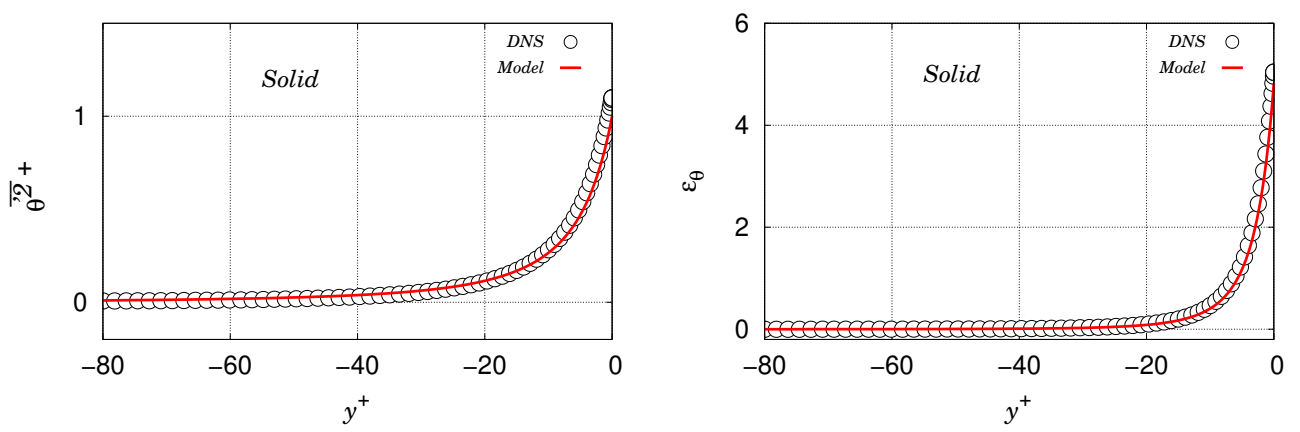

FiguRe 23. A posteriori temperature variance $\overline{\theta^{\prime 2}}$ (left) and its dissipation rate $\varepsilon_{\theta}$ (right) in the solid part for Conjugate Heat Transfer (CHT).
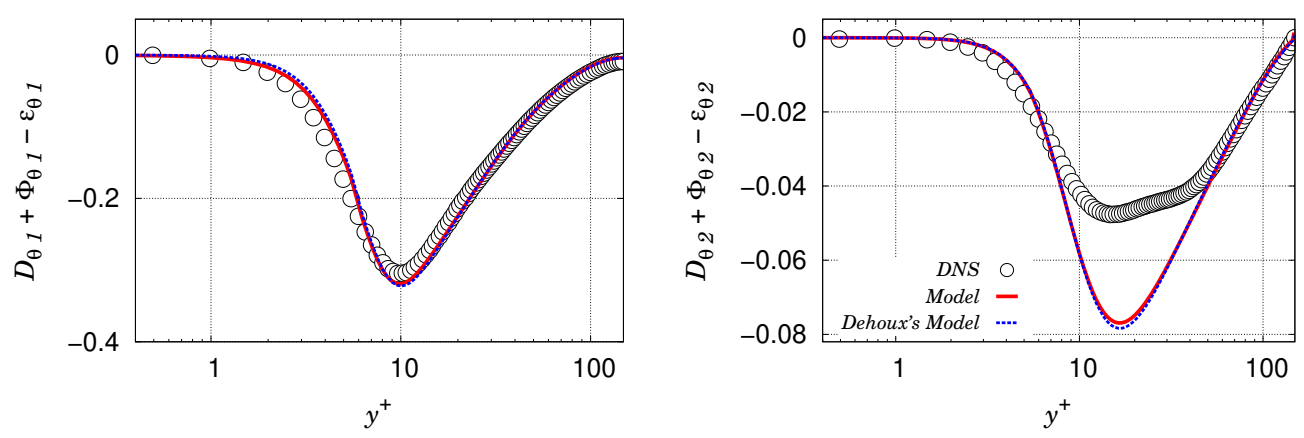

Figure 24. The near-wall balance $D_{\theta i}^{\nu+\kappa}+\phi_{\theta i}-\varepsilon_{\theta i}$ obtained in full computations for CHT compared to DNS results from Flageul et al. (2015). Left: stream-wise component; Right: wall-normal component.

heat flux components in the near-wall region. Surprisingly, Dehoux's model also provides good predictions in the near-wall region for CHT (the same result is observed in the case of an imposed temperature, which is not shown here). These results are in apparent contradiction with the asymptotic analysis and the a priori tests performed in section 3.3 , since the stream-wise component of the sum of the three terms was shown to tend to infinity at the wall. However, it is a perfect example of error compensation. Considering the different terms appearing in this balance separately for the stream-wise direction in the case of CHT (the same trend is obtained with an imposed heat flux), Fig. 25 shows that, in contrast with the new model, Dehoux's model exhibits a wrong asymptotic behavior for the dissipation term and this is compensated by a strong overestimation of molecular diffusion in the near-wall region, leading to compensation of errors.

Finally, Figs. 26, 27 and 28 show the predicted turbulent heat flux obtained with the new model and Dehoux's model against the DNS data of Flageul et al. (2015), which dictates the behavior of the mean temperature. Both the wall-normal and the streamwise components of the heat flux are correctly predicted by the new model for all the thermal boundary conditions in the regions near the wall and far from the wall. In the buffer layer, the peak of $\overline{u^{\prime} \theta^{\prime}}$ is underestimated in all the cases: as mentioned above, the elliptic blending strategy is designed to impose the correct behavior in the viscous sublayer, but is not sufficient to fully account for the complex evolution of the different terms in the buffer layer. The aforementioned error compensation in Dehoux's model 

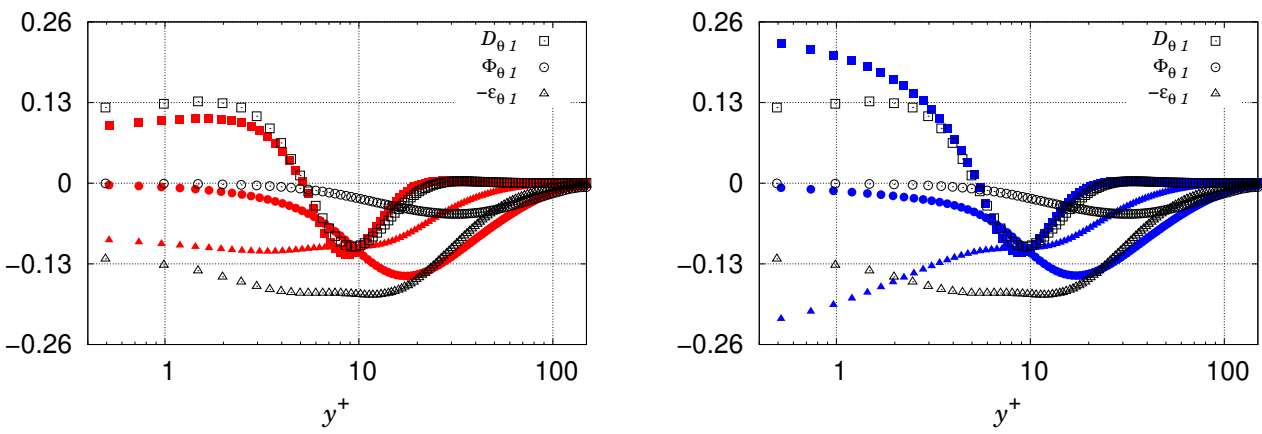

Figure 25. The dominant terms in the budgets of the stream-wise heat flux component for an Conjugate Heat Transfer (CHT). Filled symbols stand for the model; empty symbols represent the DNS data. Left: New model; Right: Dehoux's model
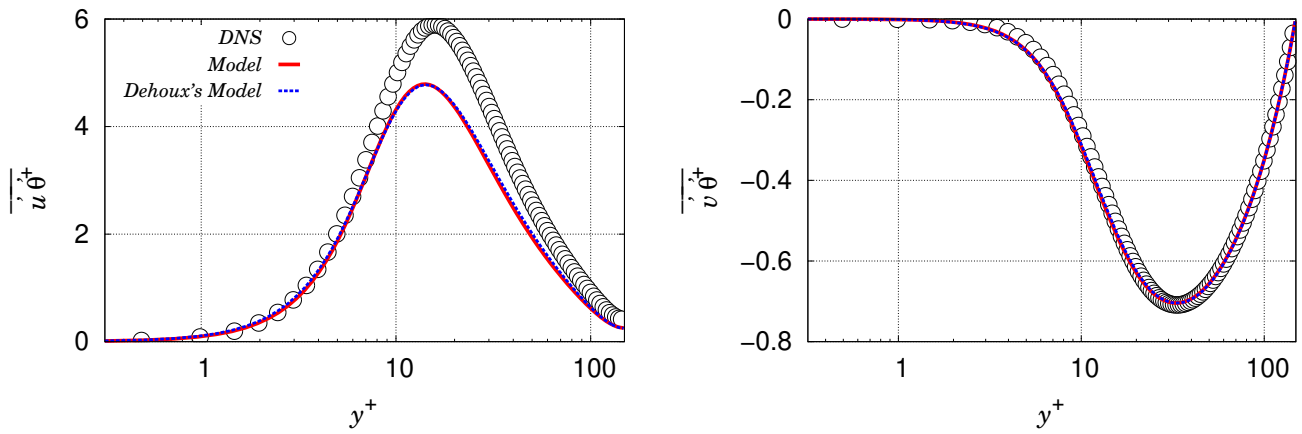

Figure 26. Predicted turbulent heat flux with an imposed temperature at the wall. Left: tangential component; Right: wall-normal component.
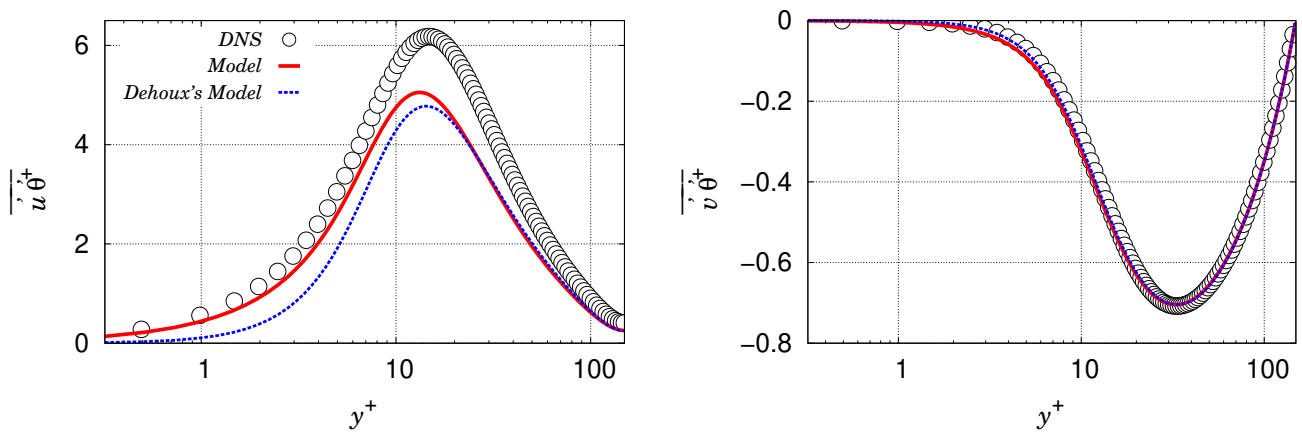

Figure 27. Predicted turbulent heat flux with an imposed heat flux at the wall. Left: tangential component; Right: wall-normal component.

makes it possible to obtain acceptable results, although the discrepancies are significant for the stream-wise component in the case of an imposed heat flux, and, to a lesser extent, for CHT. This appears as a minor issue in the present channel flow case, since the stream-wise heat flux $\overline{u^{\prime} \theta^{\prime}}$ does not affect the mean temperature profile. However, the accurate prediction of this component will gain importance in more complex flows. 

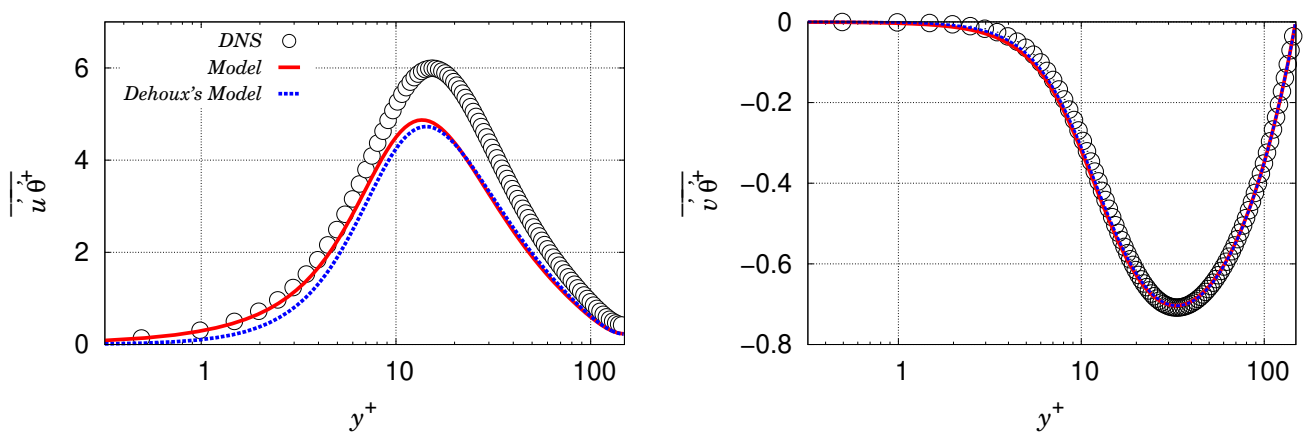

Figure 28. Predicted turbulent heat flux for Conjugate Heat Transfer (CHT). Left: tangential component; Right: wall-normal component.

\section{Conclusion}

An extended version of the DFM of Dehoux et al. (2017) is proposed in order to account for any type of thermal boundary condition at the wall. Indeed, the vanishing (imposed wall temperature) or not (imposed heat flux or CHT) of the temperature fluctuations at the wall leads to a completely different behavior of the terms of the budget of the turbulent heat flux. In order for the model to be able to adapt to the variety of boundary conditions, the key point is the use of the variable $\operatorname{Pr} / R$, i.e., the ratio of the Prandtl number to the thermal-to-mechanical time-scale ratio, which goes to unity at the wall for an imposed wall temperature and to zero otherwise. As a corollary, $R$ cannot be computed from a simple algebraic relation as in Dehoux's model, but must rather be obtained from transport equations for the temperature variance $\overline{\theta^{\prime 2}}$ and its dissipation rate $\varepsilon_{\theta}$, in such a way that the near-wall behavior of $R$ is dependent on the boundary conditions for $\overline{\theta^{\prime 2}}$ and $\varepsilon_{\theta}$.

The extended DFM is then developed based on asymptotic arguments. The function $\operatorname{Pr} / R$ is used to sensitize the scrambling term $\phi_{\theta i}$ in the transport equation for the turbulent heat flux to the thermal boundary condition, by analyzing the Taylor series expansion of the solutions of the equation. It is shown that the model for the heat flux requires an asymptotically correct behavior of the predicted thermal-to-mechanical timescale ratio, which is highly dependant on the thermal boundary condition. It is interesting to emphasize that, in the extended model, $R$ is used for what it really is, a ratio of time scales, the behaviour of which depends on the boundary conditions, and not as an artifact to avoid solving the $\varepsilon_{\theta}$ equation.

In order to ensure the correct behaviour of $R$, a new model for the dissipation rate $\varepsilon_{\theta}$ is proposed. The major term in this model is the dissipation rate $Y_{\varepsilon_{\theta}}$ : its modeling is crucial to deal with various thermal boundary conditions. To take up this challenge, in addition to $\operatorname{Pr} / R$, another key parameter is used, $\sigma_{\theta} / \varepsilon_{\theta}$, where $\sigma_{\theta}=\kappa\left(\frac{\partial \sqrt{\overline{\theta^{\prime 2}}}}{\partial y}\right)^{2}$. Indeed, $\operatorname{Pr} / R$ is not able to distinguish imposed heat flux and CHT conditions. Based on these two parameters, the dissipation term $Y_{\varepsilon_{\theta}}$ can be sensitized to the various thermal boundary conditions. A priori tests show that $\varepsilon_{\theta}$ is correctly modeled, which, in turn, leads to correct predictions of the temperature variance $\overline{\theta^{\prime 2}}$ and the thermal-to-mechanical timescale ratio $R$.

Full computations performed with the open-source solver Code_Saturne show very satisfactory results for $\varepsilon_{\theta}$ and $\overline{{\theta^{\prime}}^{2}}$ for all the thermal boundary conditions. As a consequence, satisfactory predictions in coherence with a priori considerations are obtained 
for the turbulent heat flux components and, in turn, the mean temperature in all the cases.

\section{Acknowledgements}

The $\mathrm{PhD}$ thesis of $\mathrm{G}$. Mangeon was partially supported by the ANRT (CIFRE 2017/0079) and the ANR through the project MONACO_2025 (ANR-17-CE06-0005-01 $\mathrm{ACT}$ ). The authors are indebted to C. Flageul for providing the DNS database.

\section{Appendix A. Model for the turbulent heat flux (EB-DFM)}

$$
\begin{aligned}
& \frac{\mathrm{D} \overline{u_{i}^{\prime} \theta^{\prime}}}{\mathrm{D} t}=-\overline{u_{k}^{\prime} \theta^{\prime}} \frac{\partial \overline{u_{i}}}{\partial x_{k}}-\overline{u_{i}^{\prime} u_{k}^{\prime}} \frac{\partial \bar{\theta}}{\partial x_{k}}-\beta g_{i} \overline{\theta^{\prime 2}} \\
& +\phi_{\theta i}^{*}-\varepsilon_{\theta i}+\frac{\partial}{\partial x_{k}}\left(C_{\theta} \overline{u_{k}^{\prime} u_{l}^{\prime}} \tau \frac{\partial \overline{u_{i}^{\prime} \theta^{\prime}}}{\partial x_{l}}\right) \\
& +\frac{\partial}{\partial x_{k}}\left(\frac{\kappa+\nu}{2} \frac{\partial \overline{u_{i}^{\prime} \theta^{\prime}}}{\partial x_{k}}+n_{i} n_{j} \frac{\nu-\kappa}{6} \frac{\partial \overline{u_{j}^{\prime} \theta^{\prime}}}{\partial x_{k}}\right) \\
& \varepsilon_{\theta i}=\left(1-\alpha_{\theta}\right) \varepsilon_{\theta i}^{w}+\alpha_{\theta} \varepsilon_{\theta i}^{h} \\
& \varepsilon_{\theta i}^{w}=\gamma \frac{1}{\mathcal{T}} C_{\varepsilon}\left[1+C_{w \theta}^{\varepsilon}\left(1-\alpha_{\theta}\right) \frac{P_{k}+G_{k}}{\varepsilon}\right]\left(\overline{u_{i}^{\prime} \theta^{\prime}}+\overline{u_{j}^{\prime} \theta^{\prime}} n_{i} n_{j}\right) \\
& \gamma=\frac{\sqrt{P r}}{\sqrt{R}} \\
& \varepsilon_{\theta i}^{h}=0 \\
& \phi_{\theta i}^{*}=\left(1-\alpha_{\theta}\right) \phi_{\theta i}^{w}+\alpha_{\theta} \phi_{\theta i}^{h} \\
& \phi_{\theta i}^{h}=-\frac{\sqrt{R^{h}}}{\sqrt{R} \mathcal{T}} C_{1 \theta} \overline{u_{i}^{\prime} \theta^{\prime}}+C_{2 \theta} \overline{u_{j}^{\prime} \theta^{\prime}} \frac{\partial \overline{u_{i}}}{\partial x_{j}}+C_{3 \theta} \beta g_{i} \overline{\theta^{\prime 2}} \\
& \phi_{\theta i}^{w}=-\beta \frac{1}{\mathcal{T}}\left[1+C_{w \theta}^{\phi}\left(1-\alpha_{\theta}\right) \frac{P_{k}+G_{k}}{\varepsilon}\right] \overline{u_{j}^{\prime} \theta^{\prime}} n_{i} n_{j} \\
& \beta=\frac{2}{3}+\frac{1}{3} \frac{1}{P r}+\frac{\sqrt{P r}}{\sqrt{R}} \frac{1}{3}\left(1-\frac{1}{P r}\right) \\
& \tau=\max \left(\frac{k}{\varepsilon}, C_{T}\left(\frac{\nu}{\varepsilon}\right)^{1 / 2}\right) \\
& P_{k}=-\overline{u_{i}^{\prime} u_{j}^{\prime}} \frac{\partial \overline{u_{i}}}{\partial x_{j}} ; G_{k}=-\beta \overline{u_{i}^{\prime} \theta^{\prime}} g_{i} \\
& C_{\theta}=0.22 ; C_{w \theta}^{\phi}=2 ; C_{w \theta}^{\varepsilon}=-0.3 \\
& \alpha_{\theta}-L_{\theta}^{2} \nabla^{2} \alpha_{\theta}=1
\end{aligned}
$$




$$
\begin{gathered}
L_{\theta}=2.5 L ; L=C_{L} \max \left(\frac{k^{3 / 2}}{\varepsilon}, C_{\eta} \frac{\nu^{3 / 4}}{\varepsilon^{1 / 4}}\right) \\
C_{L}=0.125 ; C_{\eta}=80 ; C_{T}=6 \\
R=\frac{\mathcal{T}}{\mathcal{T}_{\theta}} ; \mathcal{T}_{\theta}=\frac{\overline{\theta^{\prime 2}}}{2 \varepsilon_{\theta}} ; \\
\mathcal{T}=\frac{k}{\varepsilon} ; C_{\varepsilon}=\frac{1}{2}\left(1+\frac{1}{\operatorname{Pr}}\right) ; C_{1 \theta}=4.15 ; \\
C_{2 \theta}=0.3 ; C_{3 \theta}=0.5
\end{gathered}
$$

\section{Appendix B. Model for the temperature variance}

$$
\begin{gathered}
\frac{\mathrm{D} \overline{{\theta^{\prime}}^{2}}}{\mathrm{D} t}=-2 \overline{u_{k}^{\prime} \theta^{\prime}} \frac{\partial \bar{\theta}}{\partial x_{k}}-2 \varepsilon_{\theta}+\frac{\partial}{\partial x_{k}}\left(\frac{\nu}{\operatorname{Pr}} \frac{\partial \overline{\theta^{\prime 2}}}{\partial x_{k}}\right)+\frac{\partial}{\partial x_{k}}\left(C_{\theta \theta} \overline{u_{k}^{\prime} u_{l}^{\prime}} \tau \frac{\partial \overline{{\theta^{\prime}}^{2}}}{\partial x_{l}}\right) \\
C_{\theta \theta}=0.21
\end{gathered}
$$

Appendix C. Model for the dissipation rate of the temperature variance

$$
\begin{aligned}
\frac{D \varepsilon_{\theta}}{D t}= & -\frac{2}{1+P r} \varepsilon_{\theta j} \frac{\partial \bar{\theta}}{\partial x_{j}}-c_{\varepsilon_{\theta 1}} \varepsilon_{j K}^{\theta} \frac{\partial \overline{U_{j}}}{\partial x_{k}} \\
& +\kappa\left(C p_{4} \overline{u_{j}^{\prime} u_{k}^{\prime}} \frac{k}{\varepsilon} f_{R} \frac{\partial^{2} \bar{\theta}}{\partial x_{k} \partial x_{l}} \frac{\partial^{2} \bar{\theta}}{\partial x_{l} \partial x_{j}}+C p_{3} \frac{k}{\varepsilon} f_{R} \frac{\partial \overline{u_{j}^{\prime} u_{l}^{\prime}}}{\partial x_{k}} \frac{\partial \bar{\theta}}{\partial x_{l}} \frac{\partial^{2} \bar{\theta}}{\partial x_{j} \partial x_{k}}\right) \\
& +\frac{\partial}{\partial x_{j}}\left(c_{s} \overline{u_{j}^{\prime} u_{k}^{\prime}} \frac{k}{\varepsilon} \frac{\partial \varepsilon_{\theta}}{\partial x_{k}}\right)+\frac{\partial}{\partial x_{j}}\left(\kappa \frac{\partial}{\partial x_{j}} \varepsilon_{\theta}\right) \\
& +\left(1-\alpha_{\theta}\right)\left(P_{\varepsilon_{\theta}}^{w}-Y_{\varepsilon_{\theta}}^{w}\right)+\alpha_{\theta}\left(P_{\varepsilon_{\theta}}^{h}-Y_{\varepsilon_{\theta}}^{h}\right) \\
\varepsilon_{i j}^{\theta} & =\left(1-\alpha_{\theta}\right) \varepsilon_{i j}^{\theta w}+\alpha_{\theta} \varepsilon_{i j}^{\theta h},
\end{aligned}
$$

where

$$
\begin{gathered}
\varepsilon_{i j}^{\theta h}=\frac{2}{3} \varepsilon_{\theta} \delta_{i j}, \quad \varepsilon_{i j}^{\theta w}=\frac{\overline{u_{i}^{\prime} u_{j}^{\prime}}}{k} \varepsilon_{\theta} . \\
P_{\varepsilon_{\theta}}^{4}-Y_{\varepsilon_{\theta}}=\left(1-\alpha_{\theta}\right)\left(P_{\varepsilon_{\theta}}^{w}-Y_{\varepsilon_{\theta}}^{w}\right)+\alpha_{\theta}\left(P_{\varepsilon_{\theta}}^{h}-Y_{\varepsilon_{\theta}}^{h}\right), \\
P_{\varepsilon_{\theta}}^{h}=c_{\varepsilon_{\theta}}^{h 1} \frac{\varepsilon_{\theta}}{k} P_{k}+c_{\varepsilon_{\theta}}^{h 2} \frac{\varepsilon}{k} P_{\theta}, \\
Y_{\varepsilon_{\theta}}^{h}=c_{H} \frac{\varepsilon_{\theta}}{\tau_{\theta}} .
\end{gathered}
$$




\begin{tabular}{|c|c|c|c|c|c|c|c|c|}
\hline$c_{\varepsilon_{\theta 1}}$ & $c_{\varepsilon_{\theta}}^{h 1}$ & $c_{\varepsilon_{\theta}}^{h 2}$ & $c_{\varepsilon_{\theta}}^{w 1}$ & $c_{\varepsilon_{\theta}}^{w 2}$ & $C p_{3}$ & $C p_{4}$ & $f_{R}$ & $c_{s}$ \\
\hline
\end{tabular}

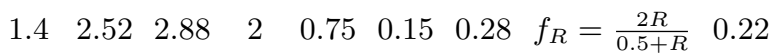

$$
\begin{aligned}
& P_{\varepsilon_{\theta}}^{w}=c_{\varepsilon_{\theta}}^{w 1}\left[1-c_{\varepsilon_{\theta}}^{w 2}\left(1-\alpha_{\theta}\right) \frac{P_{k}}{\varepsilon}\right] \frac{P_{\theta}}{\varepsilon_{\theta}} \frac{\varepsilon_{\theta}}{\sqrt{\mathcal{T} \mathcal{T}_{\theta}}} . \\
& Y_{\varepsilon_{\theta}}^{w}=\left[\left(1-\sqrt{\frac{P r}{R}}\right)\left(1-c_{w}\left(1-\alpha_{\theta}\right) \frac{P_{\theta}}{\varepsilon_{\theta}}\right)\left(c_{N}-\frac{\sigma_{\theta}}{\varepsilon_{\theta}} c_{C}\right)\right. \\
& \left.+c_{D} \sqrt{\frac{P r}{R}}\right] \frac{\varepsilon_{\theta}}{\sqrt{\tau_{\varepsilon_{\theta}} \tau_{\theta}}} \sqrt{\frac{\mathcal{T}_{\theta}}{\tau_{\theta}}} . \\
& \sigma_{\theta}=\kappa\left(\frac{\partial \sqrt{\overline{\theta^{\prime 2}}}}{\partial y}\right)^{2} \\
& \tau_{\theta}=\max \left(\mathcal{T}_{\theta}, C_{T_{\theta}} \sqrt{\frac{\nu}{\varepsilon}} \frac{1}{\operatorname{Pr}}\right) \text {, } \\
& \tau_{\varepsilon_{\theta}}=\max \left(\mathcal{T}, C_{\mathcal{T}} \sqrt{\frac{\nu}{\varepsilon}}\right) \\
& C_{\mathcal{T}}=\left(1-\sqrt{\frac{P r}{R}}\right)\left(c_{T_{N}}+\frac{\sigma_{\theta}}{\varepsilon_{\theta}} c_{T_{C}}\right)+c_{T_{D}} \sqrt{\frac{P r}{R}} .
\end{aligned}
$$

\section{REFERENCES}

Abe, H., Kawamura, H. \& Matsuo, Y. 2004 Surface heat-flux fluctuations in a turbulent channel flow up to $R_{\tau}=1020$ with $P r=0.025$ and 0.71. Int. J. Heat Fluid Fl. 25 (3), 404-419.

Abe, K., Kondoh, T. \& Nagano, Y. 1995 A new turbulence model for predicting fluid flow and heat transfer in separating and reattaching flows-II. Thermal field calculations. Int. J. Heat Mass Tran. 38, 1467-1481.

Angelino, M., Goldstein, R.J. \& GoRi, F. 2019 Lateral edge effects on heat/mass transfer on a finite width surface within a turbulent boundary layer. Int. J. Heat Mass Tran. 138, $32-40$.

Archambeau, F., Méchitoua, N. \& Sakiz, M. 2004 Code Saturne: A Finite Volume Code for the Computation of Turbulent Incompressible flows - Industrial Applications. Int. J. 
on Finite Volume, Electronical edition: http://averoes.math.univ-paris13.fr/html ISSN 1634 (0655).

Benhamadouche, S., Afgan, I. \& Manceau, R. 2020 Numerical simulations of flow and heat transfer in a wall bounded pin matrix. Flow Turbul. Combust. 104 (1), 19-44.

Billard, F. \& Laurence, D. 2012 A robust $k-\varepsilon-v^{2} / k$ elliptic blending turbulence model applied to near-wall, separated and buoyant flows. Int. J. Heat Fluid Fl. 33 (1), 45-58.

Choi, S.-K., HAN, J.-W. \& ChOI, H.-K. 2018 Performance of second-moment differential stress and flux models for natural convection in an enclosure. Int. Commun. Heat Mass Transfer 99, 54-61.

Choi, S. K. \& Kim, S. O. 2008 Treatment of turbulent heat fluxes with the elliptic-blending second-moment closure for turbulent natural convection flows. Int. J. Heat Mass Tran. 51 (9), 2377-2388.

Craft, T., Iacovides, H. \& Uapipatanakul, S. 2010 Towards the development of RANS models for conjugate heat transfer. J. Turbul. 11, N26.

Craft, T.J., Ince, N.Z. \& Launder, B.E. 1996 Recent developments in second-moment closure for buoyancy-affected flows. Dyn. Atmos. Oceans 23 (1), 99-114.

Daly, B. J. \& Harlow, F. H. 1970 Transport equations in turbulence. Physics of Fluids 13, 2634-2649.

Dehoux, F. 2012 Modélisation statistique des écoulements turbulents en convection forcée, mixte et naturelle. PhD thesis, Université de Poitiers.

Dehoux, F., Benhamadouche, S. \& Manceau, R. 2017 An elliptic blending differential flux model for natural, mixed and forced convection. Int. J. Heat Fluid Fl. 63, 190-204.

Dovizio, D., Shams, A. \& Roelofs, F. 2019 Numerical prediction of flow and heat transfer in an infinite wire-wrapped fuel assembly. Nucl. Eng. Des. 349, 193-205.

Durbin, P. A. 1991 Near-wall turbulence closure modeling without "damping functions". Theor. Comp. Fluid Dyn. 3 (1), 1-13.

Elghobashi, S. \& Launder, B. E. 1983 Turbulent time scales and the dissipation rate of temperature variance in the thermal mixing layer. Phys. Fluids 26, 2415-2419.

Flageul, C., Benhamadouche, S., Lamballais, E. \& Laurence, D. 2015 DNS of turbulent channel flow with conjugate heat transfer: Effect of thermal boundary conditions on the second moments and budgets. Int. J. Heat Fluid Fl. 55, 34-44.

Flageul, C., Benhamadouche, S., Lamballais, E. \& Laurence, D. 2017 On the discontinuity of the dissipation rate associated with the temperature variance at the fluid-solid interface for cases with conjugate heat transfer. Int. J. Heat Mass Tran. 111, $321-328$.

Hanjalić, K. 2002 One-point closure models for buoyancy-driven turbulent flows. Annu. Rev. Fluid Mech. 34, 321-347.

HANJAlić, K. \& LAUNDER, B.E. 2011 Modelling Turbulence in Engineering and the Environment. Second-Moment Routes to Closure. Cambridge University Press.

Howard, R. \& Serre, E. 2015 Large-eddy simulation in a mixing tee junction: High-order turbulent statistics analysis. Int. J. Heat Fluid Fl. 51, 65-77.

HowARD, R. \& SERRE, E. 2017 Large eddy simulation in code_saturne of thermal mixing in a T-junction with brass walls. Int. J. Heat Fluid Fl. 63, 119-127.

Jones, W.P. \& Musonge, P. 1988 Closure of the Reynolds stress and scalar flux equations. Phys. Fluids 31, 3589-3604.

Kasagi, N., Kasagi, N. \& Kuroda, A. 1992 Direct numerical simulation of passive scalar field in a turbulent channel flow. J. Heat Transf. 114, 598-606.

Kasagi, N., Kuroda, A. \& Hirata, M. 1989 Numerical investigation of near-wall turbulent heat transfer taking into account the unsteady heat conduction in the solid wall. J. Heat Transf. 111, 385-392.

LAUNDER, B. E. 1988 On the computation of convective heat transfer in complex turbulent flows. J. Heat Transf. 110, 1112-1128.

MANCEAU, R. 2015 Recent progress in the development of the elliptic blending Reynolds-stress model. Int. J. Heat Fluid Fl. 51, 195-220.

Manceau, R. \& HANJAlić, K. 2002 Elliptic blending model: A new near-wall Reynolds-stress turbulence closure. Phys. Fluids 14 (2), 744-754. 
Manceau, R., Parneix, S. \& Laurence, D. 2000 Turbulent heat transfer predictions using the $v^{2}-f$ model on unstructured meshes. Int. J. Heat Fluid Fl. 21 (3), 320-328.

Nagano, Y. 2002 Closure strategies for turbulent and transitional flows, chap. Modelling heat transfer in near-wall flows, pp. 188-247. Cambridge University Press.

Newman, G. R., Launder, B. E. \& Lumley, J. L. 1981 Modelling the behaviour of homogeneous scalar turbulence. J. Fluid Mech. 111, 217-232.

Parneix, S., Behnia, M. \& Durbin, P. A. 1998 Predictions of turbulent heat transfer in an axisymmetric jet impinging on a heated pedestal. J. Heat Transf. 120, 1-7.

Pope, S. B. 1983 Consistent modeling of scalars in turbulent flows. Phys. Fluids 26 (2), 404-408.

Shikazono, N. \& Kasagi, N. 1996 Second-moment closure for turbulent scalar transport at various Prandtl numbers. Int. J. Heat Mass Tran. 39 (14), 2977-2987.

Shin, J. K., An, J. S., Choi, Y. D., Kıм, Y. C. \& Kiм, M. S. 2008 Elliptic relaxation second moment closure for the turbulent heat fluxes. J. Turbul. 9, 1-29.

Sommer, T. P., So, R. M. C. \& Zhang, H. S. 1994 Heat transfer modeling and the assumption of zero wall temperature fluctuations. J. Heat Transf. 116 (4), 855-863.

Spalding, D.B. 1971 Concentration fluctuations in a round turbulent free jet. Chem. Eng. Sci. 26 (1), 95-107.

Tiselj, I., Bergant, R., Mavko, B., Bajsić, I. \& Hetsroni, G 2001 DNS of turbulent heat transfer in channel flow with heat conduction in the solid wall. J. Heat Transf. 123, 849-857.

YAng, G., IAcovides, H., CRAft, T. \& Apsley, D. 2019 RANS modelling for temperature variance in conjugate heat transfer. In Proc. 5th World congress on Mechanical, Chemical, and Material Engineering, Lisbon, Portugal.

Zeman, O. \& Lumley, J. L. 1976 Modeling buoyancy driven mixed layers. J. Atmos. Sci. 33, $1974-1988$. 